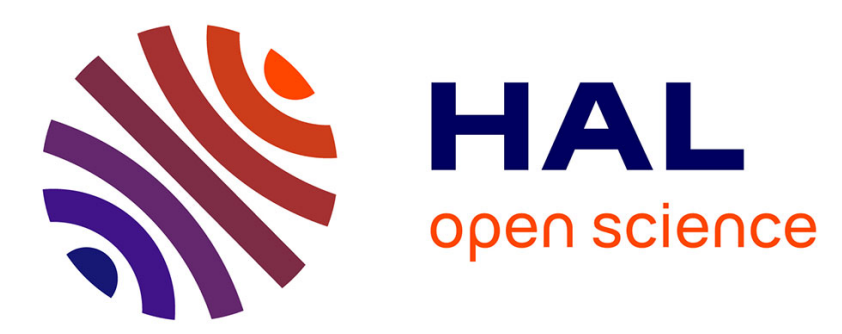

\title{
Long-Time Asymptotics of Navier-Stokes and Vorticity Equations in a Three-Dimensional Layer
}

\author{
Violaine Roussier-Michon
}

\section{To cite this version:}

Violaine Roussier-Michon. Long-Time Asymptotics of Navier-Stokes and Vorticity Equations in a Three-Dimensional Layer. Communications in Partial Differential Equations, 2004, 29 (9-10), pp.15551605. 10.1081/pde-200037768 . hal-01952337

\section{HAL Id: hal-01952337 https://hal.science/hal-01952337}

Submitted on 4 Jan 2019

HAL is a multi-disciplinary open access archive for the deposit and dissemination of scientific research documents, whether they are published or not. The documents may come from teaching and research institutions in France or abroad, or from public or private research centers.
L'archive ouverte pluridisciplinaire HAL, est destinée au dépôt et à la diffusion de documents scientifiques de niveau recherche, publiés ou non, émanant des établissements d'enseignement et de recherche français ou étrangers, des laboratoires publics ou privés. 


\title{
Long-Time Asymptotics of Navier-Stokes and Vorticity Equations in a three-dimensional Layer
}

\author{
Violaine Roussier \\ Département de mathématique, Université de Paris-Sud \\ Bat 425, F-91405 Orsay Cedex, France \\ Violaine.Roussier@math.u-psud.fr
}

May 23, 2007

Résumé: On étudie le comportement pour les grands temps des solutions de l'équation de Navier-Stokes dans la bande $\mathbf{R}^{2} \times(0,1)$. Après reformulation du problème à l'aide de variables auto-similaires, on calcule un développement asymptotique en temps de la vorticité jusqu'au second ordre, en supposant que la vorticité initiale est suffisamment petite et décroît de manire polynômiale à l'infini. Dans un deuxième temps, sans cette hypothèse de petitesse sur la donnée initiale, on prouve que, de nouveau, le comportement asymptotique des solutions globales est régi par l'équation de Navier-Stokes bidimensionnelle. En particulier, on montre que de telles solutions convergent vers le tourbillon d'Oseen.

Abstract: We study the long-time behavior of solutions of the Navier-Stokes equation in $\mathbf{R}^{2} \times(0,1)$. After introducing self-similar variables, we compute the long-time asymptotics of the vorticity up to second order, assuming that the initial vorticity is sufficiently small and has polynomial decay at infinity. Afterwards, we relax this smallness assumption and we prove again that the long-time behavior of global bounded solutions is governed by the two-dimensional NavierStokes equation. In particular, we show that solutions converge towards Oseen vortices.

Keywords: Navier-Stokes equation, long-time asymptotics, three dimensional layer, self-similar variables.

AMS classification codes (2000): 35B40, 35Q30, 35G10, 76D05

\section{Introduction}

We consider the motion of an incompressible viscous fluid filling a three dimensional layer $\mathbf{R}^{2} \times(0, L)$ where $L$ is a given length scale (for example, the depth of ocean). We denote by $x=\left(x_{1}, x_{2}\right) \in \mathbf{R}^{2}$ the horizontal variable and by $z \in(0, L)$ the vertical coordinate. If no external force is applied, the velocity field $u=\left(u_{1}, u_{2}, u_{3}\right)^{T}$ of the fluid is given by the Navier-Stokes equation

$$
\partial_{t} u+(u \cdot \nabla) u=\nu \Delta u-\frac{1}{\rho} \nabla p, \quad \operatorname{div} u=0,
$$


where $\rho$ is the density of the fluid, $\nu$ the kinematic viscosity and $p$ the pressure field. Replacing $x, z, t, u, p$ with the dimensionless quantities

$$
\frac{x}{L}, \frac{z}{L}, \frac{\nu t}{L^{2}}, \frac{L u}{\nu}, \frac{L^{2} p}{\rho \nu^{2}},
$$

equation (1) is transformed into

$$
\partial_{t} u+(u \cdot \nabla) u=\Delta u-\nabla p, \quad \operatorname{div} u=0,
$$

where $u=u(x, z, t) \in \mathbf{R}^{3}, p=p(x, z, t) \in \mathbf{R},(x, z, t) \in \mathbf{R}^{2} \times(0,1) \times \mathbf{R}^{+}$. We supplement (2) with the initial condition

$$
u(x, z, 0)=u_{0}(x, z), \quad(x, z) \in \mathbf{R}^{2} \times(0,1) .
$$

As no external force is applied, the velocity $u$ and the pressure $p$ are expected to converge to an equilibrium. Studying this asymptotic behavior is the aim of the present article.

As far as the three-dimensional Navier-Stokes equation is concerned, there have been numerous studies in the recent past years to precise the asymptotic decay in time of global solutions. Let us quote in particular the papers of M.E. Schonbek [14], [15], [16], M. Wiegner [20], A. Carpio [1], [2], and more recently of T. Miyakawa and M.E. Schonbek [10], Th. Gallay and C.E. Wayne [6]. In the present work, we show how the methods developped in [6] can be adapted to the case of a three-dimensional layer.

The previous works in a three-dimensional layer mostly deal with rotating fluids, namely with equation (2) where an external Coriolis force is added. Several articles have been written by J.Y. Chemin, B. Desjardins, I. Gallagher and E. Grenier [3] where they show that the asymptotics (when the rotation goes to infinity) are driven by a two-dimensional Navier-Stokes equation. As we shall see, the long-time behavior of (2) is also governed by the two-dimensional Navier-Stokes equation studied in [5].

We are therefore interested in the asymptotic behavior of the two dimensional Navier-Stokes equation. Let us quote three important papers which show with different methods that the first order asymptotics are driven by Oseen vortices. We recall that Oseen Vortices are particular solutions of the twodimensional Navier-Stokes equation given by

$$
u^{G}(x, t)=\frac{\alpha}{2 \pi}\left(\frac{e^{-\frac{|x|^{2}}{4 t}}-1}{|x|^{2}}\right)\left(x_{2},-x_{1}\right)^{T}, \quad \alpha \in \mathbf{R}, \quad x \in \mathbf{R}^{2}
$$

whose associated flow goes along circles. The curl of $u^{G}$ denoted by $G(x, t)=$ $\frac{\alpha}{4 \pi t} e^{-|x|^{2} / 4 t}$ is nothing else but the Gauss kernel or the fundamental solution of the heat equation. We will study more precisely those fundamental solutions later on in this paper. Y. Giga and T. Kambe [4] show the stability of the Gauss kernel with estimates on the integral equation. Highlighting the fundamental role of the heat equation inside the Navier-Stokes equation, they deal with the difference $u-u^{G}$ so that non-linear terms can be seen as perturbation ones provided the initial data is small. Their method also uses a decay estimate obtained by Y. Giga, T. Miyakawa and H. Osada. A. Carpio [1] proves the convergence to the fundamental solution of the heat equation with rescaling 
methods. Under the assumption that the fundamental solution of the twodimensional vorticity equation with initial data $\alpha \delta$ is unique, she constructs a family of initial data $\lambda u_{0}(\lambda x)$ whose vorticities converge in a weak sense to $\alpha \delta$ as $\lambda$ goes to infinity and uses the invariance of the Navier-Stokes equation under the scaling transformation

$$
u_{\lambda}(x, t)=\lambda u\left(\lambda x, \lambda^{2} t\right)
$$

to make the whole family $u_{\lambda}(t)$ converge to the Oseen Vortex. Finally, Th. Gallay and C.E. Wayne [5] construct finite dimensional invariant manifolds and use the idea that these manifolds control the long-time behavior of solutions to prove the stability of Oseen vortices. Their method also allows to compute the asymptotics of the two-dimensional Navier-Stokes equation to any order.

We supplement (2) with boundary conditions: for all $(x, z, t) \in \mathbf{R}^{3} \times \mathbf{R}^{+}$,

$$
u(x, z+1, t)=u(x, z, t) .
$$

This periodic boundary conditions (3) are not physically realistic. Nevertheless, space periodic flows are of interest in the study of homogeneous turbulence and, from the mathematical point of view, periodic boundary conditions enable us to solve functional analysis problems with the use of Fourier transformation (see $[18])$.

Although Dirichlet boundary conditions would be more realistic, they are of less interest in our case as the solutions converge exponentially fast to zero. The asymptotic behavior observed in the periodic case and the formation of Oseen vortices do not occur with the Dirichlet boundary conditions. Indeed, as we shall see, the solutions of (2) converge exponentially fast towards the two-dimensional Navier-Stokes equation. Asymptotically, the solution of (2) is thus independent of $z$ and must satisfy Dirichlet boundary conditions. This implies that such a solution of (2) together with Dirichlet conditions converges exponentially fast towards zero.

Alternatively, we will also consider stress-free boundary conditions. In this case, the force applied by the boundary on the fluid is normal to the surface and there is no shearing stress, see [19]. The mathematical translation of this situation reads for all $(x, t) \in \mathbf{R}^{2} \times \mathbf{R}^{+}$,

$$
\begin{gathered}
\frac{\partial u_{1}}{\partial z}(x, 0, t)=\frac{\partial u_{1}}{\partial z}(x, 1, t)=0 \\
\frac{\partial u_{2}}{\partial z}(x, 0, t)=\frac{\partial u_{2}}{\partial z}(x, 1, t)=0 \\
u_{3}(x, 0, t)=u_{3}(x, 1, t)=0 .
\end{gathered}
$$

In this paper, we use the vorticity formulation to study the long-time behavior of solutions of the Navier-Stokes equation (2). Setting $\omega=\operatorname{rot} u$, equation (2) is transformed into

$$
\partial_{t} \omega+(u \cdot \nabla) \omega-(\omega \cdot \nabla) u=\Delta \omega, \quad \operatorname{div} \omega=0,
$$

together with the initial condition

$$
\omega(x, z, 0)=\omega_{0}(x, z)=\operatorname{rot} u_{0}(x, z) .
$$


The velocity field $u$ can be reconstructed from $\omega$ via the Biot-Savart law (see appendix A). Boundary conditions can also be expressed in terms of the vorticity. Periodic conditions read for all $(x, z, t) \in \mathbf{R}^{3} \times \mathbf{R}^{+}$,

$$
\omega(x, z+1, t)=\omega(x, z, t)
$$

and stress-free conditions can be written for all $(x, t) \in \mathbf{R}^{2} \times \mathbf{R}^{+}$as

$$
\begin{aligned}
\omega_{1}(x, 0, t) & =\omega_{1}(x, 1, t)=0 \\
\omega_{2}(x, 0, t) & =\omega_{2}(x, 1, t)=0 \\
\frac{\partial \omega_{3}}{\partial z}(x, 0, t) & =\frac{\partial \omega_{3}}{\partial z}(x, 1, t)=0 .
\end{aligned}
$$

Although (2) and (5) are equivalent in some spaces (see [9]), we believe it is more convenient to compute long-time asymptotics in the vorticity formulation. Indeed, it has been shown, for instance by Wiegner in [20], that the decay rate in time of the velocity $u(t)$ is governed by the spacial decay rate of the initial data $u_{0}$. However, this spacial decay is not preserved under the evolution defined by (2) and $\left\{u_{0} \in L^{2}\left(\mathbf{R}^{2} \times(0,1)\right)^{3} \mid(1+|x|) u_{0} \in L^{1}\left(\mathbf{R}^{2} \times(0,1)\right)^{3}\right\}$ for instance is not an invariant set of initial data. On the other hand, the evolution of the vorticity (5) is not affected by this disadvantage. If $(1+|x|)^{m} \omega_{0} \in L^{2}\left(\mathbf{R}^{2} \times(0,1)\right)^{3}$ for some $m \geq 0$, then the solution $\omega(t)$ of (5), whenever it exists, satisfies $(1+|x|)^{m} \omega(t) \in L^{2}\left(\mathbf{R}^{2} \times(0,1)\right)^{3}$ for all $t \geq 0$. The spatial decay rate of the vorticity $\omega$ is preserved under the evolution defined by (5). Thus, we believe it is more convenient to use the vorticity formulation of the Navier-Stokes equation to compute the long-time asymptotics of the solutions.

In the first three sections of this paper, we assume $\omega(t)$ is small and decreases sufficiently fast as $|x|$ goes to infinity for all $t \geq 0$. The first property allows to deal with global bounded solutions of (5) and the second one is very helpful to study long-time asymptotics.

To actually compute the asymptotics, we use methods of infinite dynamical systems and spectral projections to reduce the study of (5) to the one of a finite number of ODE's. This idea has been developped by Th. Gallay and C.E. Wayne in [5] when building invariant manifolds to derive the long-time behavior of the vorticity. However, if we linearize equation (5) around the zero solution, the linearised equation has continuous spectrum all the way from minus infinity to zero and it is not clear how to build such manifolds. The usual idea for parabolic equations is then to express the vorticity $\omega(x, z, t)$ in terms of self-similar variables $(\xi, z, \tau)$ defined by $\xi=x / \sqrt{1+t}, \tau=\log (1+t)$, see $(7)$ below. As the scaling in time has been blown up, the rescaled linearised operator has remarkable spectral properties in weighted Lebesgue spaces that we use to compute the asymptotics of $\omega$. Indeed, we find as in [5] that the asymptotics are governed by $\bar{R} w$, the projection of the rescaled vorticity $w$ onto $z$-independent functions. Moreover, $\bar{R} w$ satisfies an evolution equation whose operator has a countable set of real, isolated eigenvalues with finite multiplicities. The essential spectrum can be pushed arbitrarily far away into the left-half plane by choosing appropriate function spaces (i.e. spatial decay rate of the vorticity). Thus, the long-time asymptotics in a neigborhood of the origin are determined, up to second order, by a finite system of ordinary differential equations.

In section 1, we prove the existence and uniqueness of global bounded solutions of the vorticity equation (5) with periodic boundary conditions in a 
neighborhood of the origin. Section 2 is devoted to the first order asymptotics. Under appropriate conditions, we show that

$$
\omega(x, z, t) \sim \frac{\alpha}{1+t} \mathbf{G}\left(\frac{x}{\sqrt{1+t}}\right), \mathbf{G}(\xi)=\frac{1}{4 \pi}\left(\begin{array}{l}
0 \\
0 \\
e^{-|\xi|^{2} / 4}
\end{array}\right)
$$

as $t$ goes to infinity, where $\alpha$ is a real coefficient which can be easily computed from the initial data. Notice that $\mathbf{G}$ is independent of $z$ and the corresponding velocity field obtained from the Biot-Savart law is horizontal, i.e. the third coordinate $u_{3}$ is zero. This velocity field is called Oseen vortex and also governs the long-time asymptotics of the two-dimensional Navier-Stokes equation (see $[1],[4],[5])$. In section 3 , we give a higher order asymptotic expansion of $\omega$ in case $\alpha=0$. This case represents the velocity of finite energy. We prove in this situation that the long-time behavior of the velocity field is two-dimensional (i.e. does not depend on $z$ ) but not horizontal (i.e. $u_{3}$ is not trivially equal to zero). Actually, we show that under appropriate conditions

$$
\omega(x, z, t) \sim \sum_{i=1}^{3} \frac{\beta_{i}}{(1+t)^{\frac{3}{2}}} \mathbf{F}_{i}\left(\frac{x}{\sqrt{1+t}}\right)
$$

when $t$ goes to infinity, where $(\beta)_{i=1, \ldots, 3}$ are real coefficients computed easily from the initial data. The vectors $\left(\mathbf{F}_{1}, \mathbf{F}_{2}, \mathbf{F}_{3}\right)$ made of derivatives of $\mathbf{G}$ are linearly independent. The three of them are two-dimensional but only $F_{1}$ and $F_{2}$ correspond to horizontal velocities. The velocity obtained from $F_{3}$ has nontrivial coordinate $u_{3}$.

The methods developped in these two sections 2 and 3 are very general and could be applied to compute the asymptotics of (5) up to any order, as soon as the spectral properties of the rescaled linearised operator mentioned above are well-known.

So far, our results concern small solutions only. In section 4, we show how they can be extended to all global bounded solutions of (5). Following [7], we relax the smallness assumption on the vorticity and compute with different methods the asymptotics of the vorticity in the same weighted spaces. Using the $\omega$-limit set of a trajectory, Lyapunov function and LaSalle's principle, we show once more that the asymptotics are governed by the $z$-independent part of the vorticity. More precisely, we prove that the velocity converges to Oseen vortices.

In section 5, we prove analogous results in the case of stress-free boundary conditions. We show that the long-time behavior of the velocity is twodimensional and horizontal. In particular, $\omega(t)$ behaves, when $t$ goes to infinity, as $\left(0,0, \omega_{2 D}\right)^{T}$, where $\omega_{2 D}$ is the solution of the two-dimensional vorticity equation studied in [5].

Finally, appendix A deals with the Biot-Savart laws in a three-dimensional layer and contains useful estimates of the velocity field in terms of the vorticity in weighted Lebesgue spaces. Appendix B is a generalisation of the study carried out in [5] on the spectrum of the two-dimensional operator $\mathcal{L}$ which governs the asymptotics of our three-dimensional equation. Next, appendix $\mathrm{C}$ describes the properties of generator $S(\tau, \sigma)$ of the evolution equation satisfied by the rescaled vorticity $w$. We compute useful estimates on $\partial^{\alpha} S(\tau, \sigma)$ in weighted Lebesgue 
spaces. Finally, appendix D gives some technical bounds on series and integrals used throughout this paper.

Notations: Throughout the paper, we denote by $\|\cdot\|_{Z}$ the norm in the Banach space $Z$ and by |.| the usual euclidean norm in $\mathbf{R}^{n}$. For any $p \in[1,+\infty]$, if $f \in L^{p}\left(\mathbf{R}^{2} \times(0,1)\right)^{3}$, we set $\|f\|_{L^{p}\left(\mathbf{R}^{2} \times(0,1)\right)}=\||f|\|_{L^{p}\left(\mathbf{R}^{2} \times(0,1)\right)}$. Weighted norms play an important role in this paper. We always denote $b(\xi)=\left(1+|\xi|^{2}\right)^{\frac{1}{2}}$, $\xi \in \mathbf{R}^{2}$, the weight function. For any $m \geq 0$, we set $\|f\|_{m}=\left\|b^{m} f\right\|_{L^{2}\left(\mathbf{R}^{2} \times(0,1)\right)}$. If $f \in \mathcal{C}^{0}\left([0, T] ; L^{p}\left(\mathbf{R}^{2} \times(0,1)\right)^{3}\right)$, we often write $f(\tau)$ to denote the map $(\xi, z) \mapsto$ $f(\xi, z, \tau)$. Finally, we denote by $C$ a generic positive constant, which may differ from place to place, even in the same chain of inequalities.

Acknowledgments: I would like to thank Thierry Gallay for all his help and suggestions regarding this work. I also thank Isabelle Gallagher for stimulating discussions.

\section{The Cauchy problem for the vorticity equa- tion}

In this section, we describe existence and uniqueness results for solutions of the vorticity equation (5). As stressed in the introduction, our approach is to study the behavior of solutions of (5) and then to derive information about the solutions of the Navier-Stokes equation as a corollary.

In $\mathbf{R}^{2} \times(0,1)$, the vorticity equation is

$$
\partial_{t} \omega+(u \cdot \nabla) \omega-(\omega \cdot \nabla) u=\Delta \omega, \quad \operatorname{div} \omega=0
$$

where $\omega=\omega(x, z, t) \in \mathbf{R}^{3}$ is 1-periodic in $z,(x, z, t) \in \mathbf{R}^{2} \times(0,1) \times \mathbf{R}^{+}$and the velocity field $u$ is defined in terms of the vorticity via the Biot-Savart law (see appendix A).

As our analysis of the long-time asymptotics of (5) depends on rewriting the equations in terms of scaling variables, we deal with the Cauchy problem in the new variables

$$
\xi=\frac{x}{\sqrt{1+t}}, \tau=\log (1+t) .
$$

As no scaling of type $z \mapsto \lambda z$ preserves the domain $(0,1)$, the third coordinate $z$ remains unchanged. If $\omega(x, z, t)$ is a solution of (5) and $u$ the corresponding velocity field, we introduce new functions $w(\xi, z, \tau)$ and $v(\xi, z, \tau)$ by

$$
\begin{aligned}
& \omega(x, z, t)=\frac{1}{1+t} w\left(\frac{x}{\sqrt{1+t}}, z, \log (1+t)\right), \\
& u(x, z, t)=\frac{1}{\sqrt{1+t}} v\left(\frac{x}{\sqrt{1+t}}, z, \log (1+t)\right) .
\end{aligned}
$$

As the transformation is time-dependent for the first two coordinates $\xi \in \mathbf{R}^{2}$, the divergence operator becomes a time-dependent operator. Namely,

$$
\operatorname{div} \omega(t)=0 \text { for any } t \geq 0 \Leftrightarrow \operatorname{div}_{\tau} w(\tau)=0 \text { for any } \tau \geq 0,
$$

where

$$
\operatorname{div}_{\tau} w(\tau)=\nabla_{\tau} \cdot w=\nabla_{\xi} \cdot w_{\xi}+e^{\frac{\tau}{2}} \partial_{z} w_{z},
$$


and

$$
\begin{gathered}
w_{\xi}=\left(w_{1}, w_{2}\right)^{T}, \quad \nabla_{\xi}=\left(\partial_{\xi_{1}}, \partial_{\xi_{2}}\right)^{T}, \\
w=\left(\begin{array}{c}
w_{\xi} \\
w_{z}
\end{array}\right), \quad \nabla_{\tau}=\left(\begin{array}{c}
\nabla_{\xi} \\
e^{\frac{\tau}{2}} \partial_{z}
\end{array}\right) .
\end{gathered}
$$

Using the same notations, notice that the relation between $w$ and $v$ reads

$$
w(\tau)=\operatorname{rot}_{\tau} v(\tau)=\nabla_{\tau} \wedge v(\tau), \quad \tau \geq 0 .
$$

Then, $w$ satisfies the evolution equation

$$
\partial_{\tau} w=\Lambda(\tau) w+N(w)(\tau), \quad \operatorname{div}_{\tau} w(\tau)=0,
$$

where

$$
\begin{aligned}
\Lambda(\tau) & =\mathcal{L}+e^{\tau} \partial_{z}^{2} \\
\mathcal{L} & =\Delta_{\xi}+\frac{1}{2} \xi \cdot \nabla_{\xi}+1 \\
N(w)(\tau) & =\left(w \cdot \nabla_{\tau}\right) v-\left(v \cdot \nabla_{\tau}\right) w \\
& =\left(w_{\xi} \cdot \nabla_{\xi}\right) v-\left(v_{\xi} \cdot \nabla_{\xi}\right) w+e^{\frac{\tau}{2}}\left(w_{z} \partial_{z} v-v_{z} \partial_{z} w\right)
\end{aligned}
$$

and the velocity field $v$ is given by the Biot-Savart law described in appendix A. Scaling variables have been previously used to study the evolution of the vorticity in [1], [4] and [5]. In those articles, the scaling variables are very convenient as they transformed an autonomous system into another one. Indeed, in $\mathbf{R}^{n}$, Navier-Stokes equation is invariant under the scaling transformation

$$
u(x, t) \rightarrow \lambda u\left(\lambda x, \lambda^{2} t\right), p(x, t) \rightarrow \lambda^{2} p\left(\lambda x, \lambda^{2} t\right) .
$$

In the three-dimensional layer $\mathbf{R}^{2} \times(0,1)$, this property is no more satisfied and the new system (9) in scaling variables is not autonomous. However, as stressed in the introduction, we shall prove that the asymptotics of (5) are governed by the two-dimensional Navier-Stokes equation in $\mathbf{R}^{2}$ which is autonomous.

As in the two-dimensional case [5], we shall solve the rescaled vorticity equation in weighted $L^{2}$-spaces. For any $m \geq 0$, we define the Hilbert space $L^{2}(m)$ by

$$
L^{2}(m)=\left\{f(\xi, z): \mathbf{R}^{3} \rightarrow \mathbf{R}^{3} \mid f \text { is 1-periodic in } z,\|f\|_{m}<\infty\right\}
$$

where

$$
\|f\|_{m}=\left(\int_{\mathbf{R}^{2} \times(0,1)}\left(1+|\xi|^{2}\right)^{m}|f(\xi, z)|^{2} d z d \xi\right)^{\frac{1}{2}}=\left\|b^{m} f\right\|_{L^{2}\left(\mathbf{R}^{2} \times(0,1)\right)} .
$$

On the contrary to what is usually done on Navier-Stokes equation (see R. Temam [19]), we do not include the condition of incompressibility in the definition of function spaces we use. As shown in (8), the divergence-free condition on $\omega$ becomes time-dependent in scaling variables and therefore cannot be taken into account to define $L^{2}(m)$. However, as this assumption on incompressibility is crucial, we always mention it in our various theorems.

In appendix $\mathrm{C}$, we show that the time-dependent operator $\Lambda(\tau)$ is the generator of a family of evolution operators (or evolution system) $S(\tau, \sigma)$ in $L^{2}(m)$ 
for any $m \geq 0$. Since $\partial_{i} \Lambda(\tau)=\left(\Lambda(\tau)+\frac{1}{2}\right) \partial_{i}$ for $i=1$ or 2 (where $\partial_{i}=\partial_{\xi_{i}}$ ) and $\partial_{z} \Lambda(\tau)=\Lambda(\tau) \partial_{z}$, it is clear that $\partial_{i} S(\tau, \sigma)=e^{\frac{\tau-\sigma}{2}} S(\tau, \sigma) \partial_{i}$ for all $0<\sigma<\tau$ and $i=1$ or 2. Thus, using the fact that $\operatorname{div}_{\tau} w(\tau)=\operatorname{div}_{\tau} v(\tau)=0$, we can rewrite (9) in integral form:

$$
\begin{aligned}
w_{i}(\tau)= & S(\tau, 0) w_{i}(0) \\
& +\int_{0}^{\tau}\left(\sum_{j=1}^{2} e^{-\frac{\tau-\sigma}{2}} \partial_{j} S(\tau, \sigma) M_{i j}(\sigma)+e^{\frac{\sigma}{2}} \partial_{z} S(\tau, \sigma) M_{i 3}(\sigma)\right) d \sigma
\end{aligned}
$$

where $i=1, . ., 3$ and

$$
M_{i j}=w_{j} v_{i}-v_{j} w_{i} .
$$

The main result of this section states that, if the initial data are small, (11) has global bounded solutions in $L^{2}(m)$.

Theorem 1.1 Let $m>1$. There exists $K_{0}>0$ such that, for all initial data $w_{0} \in L^{2}(m)$ with div $w_{0}=0$ and $\left\|w_{0}\right\|_{m} \leq K_{0}$, equation (11) has a unique global solution $w \in \mathcal{C}^{0}\left([0,+\infty) ; L^{2}(m)\right)$ satisfying $w(0)=w_{0}$ and for any $\tau \geq 0$, $\operatorname{div}_{\tau} w(\tau)=0$. In addition, there exists $K_{1}>0$ such that

$$
\|w(\tau)\|_{m} \leq K_{1}\left\|w_{0}\right\|_{m}, \quad \tau \geq 0
$$

Proof: Given $w_{0} \in L^{2}(m)$ with div $w_{0}=0$, we shall solve (11) in the Banach space

$$
X=\left\{w \in \mathcal{C}^{0}\left([0,+\infty) ; L^{2}(m)\right) \mid \operatorname{div}_{\tau} w(\tau)=0,\|w\|_{X}=\sup _{\tau \geq 0}\|w(\tau)\|_{m}<\infty\right\} .
$$

We first note that $\tau \mapsto S(\tau, 0) w_{0} \in X$ as by proposition C.1.(a) with $\alpha=0, q=$ $2, m>1$, there exists $C_{1}>0$ such that for any $\tau \geq 0$,

$$
\left\|S(\tau, 0) w_{0}\right\|_{m} \leq C_{1}\left\|w_{0}\right\|_{m} .
$$

Next, given $w \in \mathcal{C}^{0}\left([0,+\infty) ; L^{2}(m)\right)$, we define $F(w) \in \mathcal{C}^{0}\left([0,+\infty) ; L^{2}(m)\right)$ coordinate by coordinate. For $i=1, . ., 3$,

$$
F_{i}(w)(\tau)=\int_{0}^{\tau}\left(\sum_{j=1}^{2} e^{-\frac{\tau-\sigma}{2}} \partial_{j} S(\tau, \sigma) M_{i j}(\sigma)+e^{\frac{\sigma}{2}} \partial_{z} S(\tau, \sigma) M_{i 3}(\sigma)\right) d \sigma, \tau \geq 0 .
$$

We shall prove that $F$ maps $X$ into $X$ and that there exists $C_{2}>0$ such that

$$
\|F(w)\|_{X} \leq C_{2}\|w\|_{X}^{2}, \quad\left\|F(w)-F\left(w^{\prime}\right)\right\|_{X} \leq C_{2}\left\|w-w^{\prime}\right\|_{X}\left(\|w\|_{X}+\left\|w^{\prime}\right\|_{X}\right),
$$

for all $\left(w, w^{\prime}\right) \in X^{2}$. As is easily verified, the bounds (13) and (14) imply that the map $w \mapsto S(\tau, 0) w_{0}+F(w)$ has a unique fixed point in the ball $\{w \in$ $\left.X \mid\|w\|_{X} \leq R\right\}$ if $R<\left(2 C_{2}\right)^{-1}$ and $\left\|w_{0}\right\|_{m} \leq\left(2 C_{1}\right)^{-1} R$. Using Gronwall's lemma, it is then straightforward to show that this fixed point is actually the unique solution of (11) in the space $\mathcal{C}^{0}\left([0,+\infty) ; L^{2}(m)\right)$. Finally, since $\|w\|_{X} \leq$ $C_{1}\left\|w_{0}\right\|_{m}+C_{2}\|w\|_{X}^{2} \leq C_{1}\left\|w_{0}\right\|_{m}+\frac{1}{2}\|w\|_{X}$, the bound (12) holds with $K_{1}=2 C_{1}$. 
To prove (14), we use the bounds on $S(\tau, \sigma)$ proved in appendix C. First,

$$
\begin{aligned}
\left\|F_{i}(w)(\tau)\right\|_{m} \leq \int_{0}^{\tau} \sum_{j=1}^{2} e^{-\frac{\tau-\sigma}{2}}\left\|\partial_{j} S(\tau, \sigma) M_{i j}(\sigma)\right\|_{m} d \sigma \\
\quad+\int_{0}^{\tau} e^{\frac{\sigma}{2}}\left\|\partial_{z} S(\tau, \sigma) M_{i 3}(\sigma)\right\|_{m} d \sigma .
\end{aligned}
$$

The first integral is bounded by proposition C.1(a) with $\alpha=(1,0,0)$ or $(0,1,0)$, $q=\frac{3}{2}$ and $m>1$. The second one is bounded by proposition C.1(b) with $\alpha=(0,0,1), q=\frac{3}{2}$ and $m>1$. Then, for $i=1, . ., 3$,

$$
\begin{aligned}
\left\|F_{i}(w)(\tau)\right\|_{m} \leq & C \int_{0}^{\tau} \sum_{j=1}^{2} \frac{e^{-\frac{\tau-\sigma}{2}}}{a(\tau-\sigma)^{\frac{2}{3}} a\left(e^{\tau}-e^{\sigma}\right)^{\frac{1}{12}}}\left\|b^{m} M_{i j}(\sigma)\right\|_{L^{\frac{3}{2}}\left(\mathbf{R}^{2} \times(0,1)\right)} d \sigma \\
& +C \int_{0}^{\tau} \frac{e^{\frac{\sigma}{2}} e^{-4 \pi^{2}\left(e^{\tau}-e^{\sigma}\right)}}{a(\tau-\sigma)^{\frac{1}{6}} a\left(e^{\tau}-e^{\sigma}\right)^{\frac{7}{12}}}\left\|b^{m} M_{i 3}(\sigma)\right\|_{L^{\frac{3}{2}}\left(\mathbf{R}^{2} \times(0,1)\right)} d \sigma .
\end{aligned}
$$

As $a(\tau-\sigma) \leq a\left(e^{\tau}-e^{\sigma}\right)$, it is clear that

$$
\int_{0}^{\tau} \frac{e^{-\frac{\tau-\sigma}{2}}}{a(\tau-\sigma)^{\frac{2}{3}} a\left(e^{\tau}-e^{\sigma}\right)^{\frac{1}{12}}} d \sigma \leq \int_{0}^{\infty} \frac{e^{-u / 2}}{a(u)^{\frac{3}{4}}} d u<+\infty
$$

and by appendix D.2 with $(\alpha, \beta, \gamma, \delta)=\left(\frac{1}{2}, 0, \frac{1}{6}, \frac{7}{12}\right)$, we get

$$
\int_{0}^{\tau} \frac{e^{\frac{\sigma}{2}} e^{-4 \pi^{2}\left(e^{\tau}-e^{\sigma}\right)}}{a(\tau-\sigma)^{\frac{1}{6}} a\left(e^{\tau}-e^{\sigma}\right)^{\frac{7}{12}}} d \sigma \leq C e^{-\frac{\tau}{3}}<+\infty
$$

Then, we just need to bound $\left\|b^{m} M_{i j}\right\|_{L^{\frac{3}{2}}\left(\mathbf{R}^{2} \times(0,1)\right)}$ in terms of $\|w\|_{X}^{2}$ to get the first inequality of (14). Using Hölder's inequality, we get

$$
\left\|b^{m} w_{j} v_{i}\right\|_{L^{\frac{3}{2}}\left(\mathbf{R}^{2} \times(0,1)\right)} \leq\left\|b^{m} w_{j}\right\|_{L^{2}\left(\mathbf{R}^{2} \times(0,1)\right)}\left\|v_{i}\right\|_{L^{6}\left(\mathbf{R}^{2} \times(0,1)\right)} .
$$

Dividing $v_{i}$ into two parts as in appendix A, $v_{i}=\bar{v}_{i}+\tilde{v}_{i}$, see (42) and using the Biot-Savart laws proved in appendix A.4, we get

$$
\begin{aligned}
\left\|\bar{v}_{i}\right\|_{L^{6}\left(\mathbf{R}^{2}\right)} & \leq C\|\bar{w}\|_{L^{\frac{3}{2}}\left(\mathbf{R}^{2}\right)} \\
\left\|\tilde{v}_{i}\right\|_{L^{6}\left(\mathbf{R}^{2} \times(0,1)\right)} & \leq C\|\tilde{w}\|_{L^{2}\left(\mathbf{R}^{2} \times(0,1)\right)} .
\end{aligned}
$$

Finally, by Hlder's inequality, we have $L^{2}(m) \hookrightarrow L^{q}\left(\mathbf{R}^{2} \times(0,1)\right)$ for all $q \in[1,2]$, $m>1$, and the following estimates

$$
\|\bar{w}\|_{L^{\frac{3}{2}\left(\mathbf{R}^{2}\right)}} \leq C\|w\|_{m} \text { and }\|\tilde{w}\|_{L^{2}\left(\mathbf{R}^{2} \times(0,1)\right)} \leq C\|w\|_{m}
$$

lead to the conclusion $\left\|b^{m} w_{j} v_{i}\right\|_{L^{\frac{3}{2}}\left(\mathbf{R}^{2} \times(0,1)\right)} \leq C\|w\|_{m}^{2}$. Then, $\|F(w)\|_{X} \leq$ $C_{2}\|w\|_{X}^{2}$ and the second inequality in (14) can be proved along the same lines. The proof of theorem 1.1 is now complete.

We translate theorem 1.1 in terms of the vorticity $\omega(x, z, t)$ in the original variables: 
Corollary 1.2 Let $m>1$. There exists $\epsilon_{0}>0$ such that for all initial data $\omega_{0} \in$ $L^{2}(m)$ with div $\omega_{0}=0$ and $\left\|\omega_{0}\right\|_{m} \leq \epsilon_{0}$, equation (5) has a unique global solution $\omega \in \mathcal{C}^{0}\left([0,+\infty) ; L^{2}(m)\right)$ satisfying $\omega(0)=\omega_{0}$ and div $\omega=0$. In addition, for any $p \in[1,2]$, there exists $\epsilon_{1}>0$ such that

$$
\|\omega(t)\|_{L^{p}\left(\boldsymbol{R}^{2} \times(0,1)\right)} \leq \frac{\epsilon_{1}}{(1+t)^{1-\frac{1}{p}}}\left\|\omega_{0}\right\|_{m}, \quad t \geq 0 .
$$

Proof: First take $\epsilon_{0}=K_{0}$. If $\omega_{0} \in L^{2}(m)$ satisfies div $\omega_{0}=0$ and $\left\|\omega_{0}\right\|_{m} \leq \epsilon_{0}$, the function $w_{0}$ defined by $(7)$ is in $L^{2}(m)$ for $m>1$ and $\left\|w_{0}\right\|_{m} \leq K_{0}$. By theorem 1.1, there exists a unique solution $w \in \mathcal{C}^{0}\left([0,+\infty) ; L^{2}(m)\right)$ to $(11)$ satisfying $w(0)=w_{0}$ and for any $\tau \geq 0, \operatorname{div}_{\tau} w(\tau)=0$. Let $\omega$ be the corresponding vorticity defined by $(7)$. Then, as $\omega(t) \in L^{2}(m)$ and $L^{2}(m) \hookrightarrow L^{p}\left(\mathbf{R}^{2} \times(0,1)\right)$ for $p \in[1,2], m>1$,

$$
\begin{aligned}
\|\omega(t)\|_{L^{p}\left(\mathbf{R}^{2} \times(0,1)\right)} & =(1+t)^{-1+\frac{1}{p}}\|w(\tau)\|_{L^{p}\left(\mathbf{R}^{2} \times(0,1)\right)} \\
& \leq C(1+t)^{-1+\frac{1}{p}}\|w(\tau)\|_{m} \\
& \leq \frac{C K_{1}}{(1+t)^{1-\frac{1}{p}}}\left\|w_{0}\right\|_{m} .
\end{aligned}
$$

Taking $\epsilon_{1}=C K_{1}$ ends the proof of the corollary.

Remark: Due to the embedding $L^{2}(m) \hookrightarrow L^{p}\left(\mathbf{R}^{2} \times(0,1)\right)$ which is true for $p \in[1,2]$, the proof only holds for this range of $p$. However, due to the regularising effect, (16) holds for all $p \in[1,+\infty]$ if $t \geq 1$.

In order to compare these estimates with other known results on NavierStokes, it is worth stating the previous corollary in terms of the physical variables which appear in equation (1). Define the physical vorticity $\Omega$ by

$$
\frac{L^{2}}{\nu} \Omega\left(L x, L z, \frac{L^{2}}{\nu} t\right)=\omega(x, z, t), \quad(x, z, t) \in \mathbf{R}^{2} \times(0,1) \times \mathbf{R}^{+} .
$$

Then, $\Omega$ satisfies the vorticity equation associated with (1) and corollary 1.2 states that if $L^{\frac{1}{2}}\left\|\left(1+\frac{|x|}{L}\right)^{m} \Omega_{0}\right\|_{L^{2}\left(\mathbf{R}^{2} \times(0,1)\right)} \leq \epsilon_{0} \nu$, then

$$
L^{2-3 / p}\|\Omega(t)\|_{L^{p}\left(\mathbf{R}^{2} \times(0,1)\right)} \leq \frac{\epsilon_{1} \nu}{\left(1+\frac{\nu t}{L^{2}}\right)^{1-\frac{1}{p}}} .
$$

This last inequality clearly shows the influence of the kinematic viscosity $\nu$. In particular, the smallness assumption of the initial data required in the first three sections is, in fact, a comparison between the physical vorticity and the viscosity.

\section{First-Order Asymptotics}

In this section, we consider the behavior of small solutions of the integral equation (11) in $L^{2}(m)$ for $m>1$. In $\bar{R}\left(L^{2}(m)\right)$ where $\bar{R}$ is defined in (50), the discrete spectrum of $\mathcal{L}$ contains at least a simple isolated eigenvalue $\lambda_{0}=0$ (see appendix B.1) with eigenfunction $\mathbf{G}=(0,0, G)^{T}$ where here and in the sequel, $G$ is the gaussian function:

$$
G(\xi)=\frac{1}{4 \pi} \exp \left(-\frac{|\xi|^{2}}{4}\right), \xi \in \mathbf{R}^{2} .
$$


Let $\mathbf{v}^{G}$ denote the corresponding velocity field, satisfying $\operatorname{rot} \mathbf{v}^{G}=\mathbf{G}$. Then,

$$
\mathbf{v}^{G}(\xi)=\frac{1}{2 \pi} \frac{e^{-|\xi|^{2} / 4}-1}{|\xi|^{2}}\left(\begin{array}{c}
\xi_{2} \\
-\xi_{1} \\
0
\end{array}\right), \xi=\left(\xi_{1}, \xi_{2}\right) \in \mathbf{R}^{2}
$$

and $\left(\mathbf{v}^{G} \cdot \nabla\right) \mathbf{G}=(\mathbf{G} \cdot \nabla) \mathbf{v}^{G}=0$. As a consequence, for any $\alpha \in \mathbf{R}, w(\xi, z)=$ $\alpha \mathbf{G}(\xi)$ is a stationary solution of (9) whose velocity $\alpha \mathbf{v}^{G}$ is called Oseen Vortex. Using these notations and appendix B.3, any solution $w$ of $(11)$ in $L^{2}(m)$ for $m>1$ can be decomposed as

$$
\begin{aligned}
w(\xi, z, \tau) & =P_{0} w(\xi, z, \tau)+Q_{0} w(\xi, z, \tau)+\tilde{R} w(\xi, z, \tau) \\
& \equiv \alpha(\tau) \mathbf{G}(\xi)+q_{0}(\xi, \tau)+r(\xi, z, \tau)
\end{aligned}
$$

where the projections $P_{0}, Q_{0}, \tilde{R}$ and the coefficient $\alpha$ are defined in appendix B by $(50,54,56)$. Then, $q_{0}$ belongs to the subspace $\mathcal{W}_{0}$ of $\bar{R}\left(L^{2}(m)\right)$ defined in (55) which is also the spectral subspace associated with the strictly stable part of the spectrum of $\mathcal{L}$ in $\bar{R}\left(L^{2}(m)\right)$. In particular, $\int_{\mathbf{R}^{2}} q_{0}(\xi, \tau) d \xi=0$ for all $\tau \geq 0$. Moreover, $\int_{0}^{1} r(\xi, z, \tau) d z=0$. Notice that the notations $r$ and $\tilde{w}$ are equivalent.

As in the two-dimensional case [5], an important property of (11) is the conservation of mass:

Lemma 2.1 Assume $m>1$ and $w \in \mathcal{C}^{0}\left([0, T] ; L^{2}(m)\right)$ is a solution of (11). Then, the coefficient $\alpha$ defined in (54) is constant in time.

Proof: As $\alpha(\tau)=\int_{\mathbf{R}^{2} \times(0,1)} w_{3}(\xi, z, \tau) d \xi d z$, integrating by parts shows that

$$
\begin{aligned}
\dot{\alpha}(\tau) & =\int_{\mathbf{R}^{2} \times(0,1)}\left(\mathcal{L} w_{3}+e^{\tau} \partial_{z}^{2} w_{3}+N(w)_{3}\right) d z d \xi \\
& =\int_{\mathbf{R}^{2} \times(0,1)}\left(\nabla_{\xi} \cdot\left(\nabla_{\xi} w_{3}+\frac{1}{2} \xi w_{3}\right)-v_{3} \operatorname{div}_{\tau} w(\tau)+w_{3} \operatorname{div}_{\tau} v(\tau)\right) d z d \xi \\
& =0
\end{aligned}
$$

as $w$ and $v$ are 1-periodic in $z$ and decreasing in $\xi$ at infinity.

In particular, it follows from lemma 2.1 that $\mathcal{W}_{0}$ is invariant under the evolution defined by (11). The remainder terms $q_{0}$ and $r$ defined in (17) satisfy the equations:

$$
\begin{aligned}
\partial_{\tau} q_{0} & =\mathcal{L} q_{0}+Q_{0}(N(w)), \quad \operatorname{div} q_{0}=0, \xi \in \mathbf{R}^{2}, \tau>0 \\
\partial_{\tau} r & =\Lambda(\tau) r+\tilde{R}(N(w)), \quad \operatorname{div}_{\tau} r(\tau)=0,(\xi, z) \in \mathbf{R}^{2} \times(0,1), \tau>0
\end{aligned}
$$

where $Q_{0}$ and $\tilde{R}$ are the projections defined in (56) and (50). The main result of this section states that, if the initial data are small, the solution of (11) converges to the vorticity associated with Oseen Vortex:

Theorem 2.2 Let $0<\mu<\frac{1}{2}$ and $m>1+2 \mu$. There exists $K_{0}^{\prime}>0$ such that, for all initial data $w_{0} \in L^{2}(m)$ with div $w_{0}=0$ and $\left\|w_{0}\right\|_{m} \leq K_{0}^{\prime}$, equation (11) has a unique global solution $w \in \mathcal{C}^{0}\left([0,+\infty) ; L^{2}(m)\right)$ satisfying $w(0)=w_{0}$ and for any $\tau \geq 0$, div $\tau w(\tau)=0$. In addition, there exists $K_{2}>0$ such that

$$
\|w(\tau)-\alpha \boldsymbol{G}\|_{m} \leq K_{2} e^{-\mu \tau}\left\|w_{0}\right\|_{m}, \quad \tau \geq 0,
$$

where $\alpha=\int_{R^{2} \times(0,1)}\left(w_{0}\right)_{3}(\xi, z) d z d \xi$. 
Remark: In fact, one can show that theorem 2.2 remains true for $\mu=\frac{1}{2}$, but the proof below is limited to $\mu<\frac{1}{2}$ for technical reasons.

Proof: If $w \in \mathcal{C}^{0}\left([0, \infty) ; L^{2}(m)\right)$ is the solution of (11) given by theorem 1.1 for $K_{0}^{\prime} \leq K_{0}$ and $v$ the corresponding velocity field, we define $\alpha, q_{0}$ and $r$ as in (17). By lemma 2.1, $\alpha(\tau)=\alpha(0)=\alpha$ for all $\tau \geq 0$. To bound the remainder $q_{0}$ and $r$, we use the integral equations

$$
\begin{gathered}
q_{0}(\tau)=e^{\tau \mathcal{L}} q_{0}(0)+\int_{0}^{\tau} e^{(\tau-\sigma) \mathcal{L}} Q_{0}(N(w)(\sigma)) d \sigma, \tau \geq 0, \\
r(\tau)=S(\tau, 0) r(0)+\int_{0}^{\tau} S(\tau, \sigma) \tilde{R}(N(w)(\sigma)) d \sigma, \tau \geq 0 .
\end{gathered}
$$

We first easily prove that

$$
\left\|e^{\tau \mathcal{L}} q_{0}(0)\right\|_{m} \leq C e^{-\mu \tau}\left\|w_{0}\right\|_{m}
$$

Indeed, (22) follows from proposition B.1 with $n=0, \alpha=0, q=2$ and $\epsilon \in(0, m-1-2 \mu)$.

In the same way, by proposition C.1(b) with $\alpha=0$ and $q=2$, there exists $C>0$ such that

$$
\|S(\tau, 0) r(0)\|_{m} \leq C e^{-4 \pi^{2} e^{\tau}}\left\|w_{0}\right\|_{m} .
$$

To estimate integrals in $(20,21)$, we proceed as in the proof of inequalities (14) in theorem 1.1. However, $\|w(\tau)\|_{m}$ does not converge (in general) to zero and since we want to prove that $w(\tau)$ converges to $\alpha \mathbf{G}$, the above method is not sufficient to conclude. The right procedure is to bound the integrals in $(20,21)$ by $\left\|q_{0}\right\|_{m}+\|r\|_{m}$ which will converge to zero. Therefore, we first notice that the non-linearity $N=\left(w \cdot \nabla_{\tau}\right) v-\left(v \cdot \nabla_{\tau}\right) w$ does not contain any terms in $\alpha^{2}$. If we decompose $w$ as $\alpha \mathbf{G}+q_{0}+r$ and $v$ as $\alpha \mathbf{v}^{G}+\mathbf{v}^{q}+\mathbf{v}^{r}$ where $v^{q}$ and $v^{r}$ are the velocities associated via the Biot-Savart law to the vorticities $q_{0}$ and $r$ respectively, $N$ can be written as:

$$
\begin{aligned}
N= & \alpha^{2}\left(\left(\mathbf{G} \cdot \nabla_{\tau}\right) \mathbf{v}^{G}-\left(\mathbf{v}^{G} \cdot \nabla_{\tau}\right) \mathbf{G}\right) \\
& +\alpha\left(\left(\mathbf{G} \cdot \nabla_{\tau}\right)\left(\mathbf{v}^{q}+\mathbf{v}^{r}\right)-\left(\left(\mathbf{v}^{q}+\mathbf{v}^{r}\right) \cdot \nabla_{\tau}\right) \mathbf{G}\right) \\
& +\alpha\left(\left(\left(q_{0}+r\right) \cdot \nabla_{\tau}\right) \mathbf{v}^{G}-\left(\mathbf{v}^{G} \cdot \nabla_{\tau}\right)\left(q_{0}+r\right)\right) \\
& +\left(\left(q_{0}+r\right) \cdot \nabla_{\tau}\right)\left(\mathbf{v}^{q}+\mathbf{v}^{r}\right)-\left(\left(\mathbf{v}^{q}+\mathbf{v}^{r}\right) \cdot \nabla_{\tau}\right)\left(q_{0}+r\right) .
\end{aligned}
$$

Since $\left(\mathbf{G} \cdot \nabla_{\tau}\right) \mathbf{v}^{G}=\left(\mathbf{v}^{G} \cdot \nabla_{\tau}\right) \mathbf{G}=0, N$ depends linearly on $\alpha$. Then, for $i=1, . ., 3$,

$$
N_{i}=\sum_{j=1}^{2} \partial_{j} \hat{M}_{i j}+e^{\frac{\tau}{2}} \partial_{z} \hat{M}_{i 3}
$$

where for $(i, j) \in\{1, . ., 3\}^{2}$,

$$
\begin{aligned}
\hat{M}_{i j}= & \alpha\left(\mathbf{G}_{j}\left(\mathbf{v}_{i}^{q}+\mathbf{v}_{i}^{r}\right)-\mathbf{G}_{i}\left(\mathbf{v}_{j}^{q}+\mathbf{v}_{j}^{r}\right)+\mathbf{v}_{i}^{G}\left(q_{0 j}+r_{j}\right)-\mathbf{v}_{j}^{G}\left(q_{0 i}+r_{i}\right)\right) \\
& +\left(q_{0 j}+r_{j}\right)\left(\mathbf{v}_{i}^{q}+\mathbf{v}_{i}^{r}\right)-\left(q_{0 i}+r_{i}\right)\left(\mathbf{v}_{j}^{q}+\mathbf{v}_{j}^{r}\right) .
\end{aligned}
$$


As $\int_{\mathbf{R}^{2}} q_{0}(\xi, \tau) d \xi=\int_{0}^{1} r(\xi, z, \tau) d z=0$,

$$
\begin{aligned}
Q_{0}(N(w)(\sigma)) & =\int_{0}^{1} N(w) d z=\left(\sum_{j=1}^{2} \partial_{j} \int_{0}^{1} \hat{M}_{i j} d z\right)_{i=1, . .3} \\
\tilde{R}(N(w)(\sigma)) & =\left(\sum_{j=1}^{2}\left(\partial_{j} \hat{M}_{i j}-\partial_{j} \int_{0}^{1} \hat{M}_{i j} d z\right)+e^{\frac{\sigma}{2}} \partial_{z} \hat{M}_{i 3}\right)_{i=1, . ., 3}
\end{aligned}
$$

Easily, we find for $i=1, . ., 3$,

$$
\begin{aligned}
& e^{(\tau-\sigma) \mathcal{L}} Q_{0}(N(w))_{i}=\sum_{j=1}^{2} e^{-\frac{\tau-\sigma}{2}} e^{(\tau-\sigma) \mathcal{L}} \int_{0}^{1} \hat{M}_{i j} d z \\
& S(\tau, \sigma) \tilde{R}(N(w))_{i}=\sum_{j=1}^{2} e^{-\frac{\tau-\sigma}{2}} \partial_{j} S(\tau, \sigma) \tilde{R}\left(\hat{M}_{i j}\right)+e^{\frac{\sigma}{2}} \partial_{z} S(\tau, \sigma) \hat{M}_{i 3} .
\end{aligned}
$$

Noticing that by Jensen's inequality,

$$
\left\|b^{m} \int_{0}^{1} \hat{M}_{i j} d z\right\|_{L^{\frac{3}{2}}\left(\mathbf{R}^{2}\right)} \leq\left\|b^{m} \hat{M}_{i j}\right\|_{L^{\frac{3}{2}}\left(\mathbf{R}^{2} \times(0,1)\right)},
$$

we obtain as in theorem 1.1:

$$
\begin{gathered}
\left\|e^{(\tau-\sigma) \mathcal{L}} Q_{0}(N)\right\|_{m} \leq C \sum_{(i, j) \in I} \frac{e^{-\frac{\tau-\sigma}{2}}}{a(\tau-\sigma)^{\frac{2}{3}} a\left(e^{\tau}-e^{\sigma}\right)^{\frac{1}{12}}}\left\|b^{m} \hat{M}_{i j}\right\|_{L^{\frac{3}{2}}\left(\mathbf{R}^{2} \times(0,1)\right)} \\
\|S(\tau, \sigma) \tilde{R}(N)\|_{m} \leq C \sum_{(i, j) \in I} \frac{e^{-\frac{\tau-\sigma}{2}} e^{-4 \pi^{2}\left(e^{\tau}-e^{\sigma}\right)}}{a(\tau-\sigma)^{\frac{2}{3}} a\left(e^{\tau}-e^{\sigma}\right)^{\frac{1}{12}}}\left\|b^{m} \hat{M}_{i j}\right\|_{L^{\frac{3}{2}}\left(\mathbf{R}^{2} \times(0,1)\right)} \\
+C \sum_{i=1}^{3} \frac{e^{\frac{\sigma}{2}} e^{-4 \pi^{2}\left(e^{\tau}-e^{\sigma}\right)}}{a(\tau-\sigma)^{\frac{1}{6}} a\left(e^{\tau}-e^{\sigma}\right)^{\frac{7}{12}}}\left\|b^{m} \hat{M}_{i 3}\right\|_{L^{\frac{3}{2}}\left(\mathbf{R}^{2} \times(0,1)\right)}
\end{gathered}
$$

where $I=\{1,2,3\} \times\{1,2\}$. Next, proceeding as in theorem 1.1, by Hölder's inequality and Biot-Savart law,

$$
\begin{aligned}
\left\|b^{m} \hat{M}_{i j}\right\|_{L^{\frac{3}{2}}} \leq & \alpha\left(\left\|b^{m} \mathbf{G}_{j}\left(\mathbf{v}_{i}^{q}+\mathbf{v}_{i}^{r}\right)\right\|_{L^{\frac{3}{2}}}+\left\|b^{m} \mathbf{G}_{i}\left(\mathbf{v}_{j}^{q}+\mathbf{v}_{j}^{r}\right)\right\|_{L^{\frac{3}{2}}}\right) \\
& +\alpha\left(\left\|b^{m} \mathbf{v}_{j}^{G}\left(q_{0 i}+r_{i}\right)\right\|_{L^{\frac{3}{2}}}+\left\|b^{m} \mathbf{v}_{i}^{G}\left(q_{0 j}+r_{j}\right)\right\|_{L^{\frac{3}{2}}}\right) \\
& +\left\|b^{m}\left(q_{0 j}+r_{j}\right)\left(\mathbf{v}_{i}^{q}+\mathbf{v}_{i}^{r}\right)\right\|_{L^{\frac{3}{2}}}+\left\|b^{m}\left(q_{0 i}+r_{i}\right)\left(\mathbf{v}_{j}^{q}+\mathbf{v}_{j}^{r}\right)\right\|_{L^{\frac{3}{2}}} \\
\leq & C(w)\left(\left\|q_{0}\right\|_{m}+\|r\|_{m}\right)
\end{aligned}
$$

where $C(w)=2 C\left(2 \alpha\|\mathbf{G}\|_{m}+\left\|q_{0}+r\right\|_{m}\right)$. Then, by theorem 1.1, $|C(w)| \leq$ $C_{0}\|w\|_{m} \leq C_{0} K_{1}\left\|w_{0}\right\|_{m}$ and $|C(w)|$ can be taken as small as we want by choice of appropriate initial data $w_{0}$.

Finally, denoting $f(\tau)=e^{\mu \tau}\left(\left\|q_{0}(\tau)\right\|_{m}+\|r(\tau)\|_{m}\right)$, we get

$$
\begin{aligned}
f(\tau) & \leq C\left\|w_{0}\right\|_{m} \\
& +C C(w) \int_{0}^{\tau}\left(\frac{e^{\left(\mu-\frac{1}{2}\right)(\tau-\sigma)}}{a(\tau-\sigma)^{\frac{2}{3}} a\left(e^{\tau}-e^{\sigma}\right)^{\frac{1}{12}}}+\frac{e^{\frac{\sigma}{2}} e^{\mu(\tau-\sigma)} e^{-4 \pi^{2}\left(e^{\tau}-e^{\sigma}\right)}}{a(\tau-\sigma)^{\frac{1}{6}} a\left(e^{\tau}-e^{\sigma}\right)^{\frac{7}{12}}}\right) f(\sigma) d \sigma .
\end{aligned}
$$


As $0<\mu<1 / 2$ and $m>1+2 \mu$, the first part of the integral is bounded as in (15) and the second one by appendix D.2 with $(\alpha, \beta, \gamma, \delta)=\left(\frac{1}{2}, \mu, \frac{1}{6}, \frac{7}{12}\right)$ there exist positive constants $C_{1}, C_{2}$ such that for any $T>0$,

$$
\|f\|_{L^{\infty}(0, T)} \leq C_{1}\left\|w_{0}\right\|_{m}+C_{2} C(w)\|f\|_{L^{\infty}(0, T)} .
$$

Taking $K_{0}^{\prime}>0$ such that $\left|C_{2} C(w)\right| \leq C_{0} C_{2} K_{1} K_{0}^{\prime} \leq 1 / 2$, we get

$$
\|f\|_{L^{\infty}(0, T)} \leq 2 C_{1}\left\|w_{0}\right\|_{m} .
$$

Then, for any $\tau \geq 0$,

$$
\|w(\tau)-\alpha \mathbf{G}\|_{m} \leq 2 C_{1}\left\|w_{0}\right\|_{m} e^{-\mu \tau} .
$$

Then $K_{2}=2 C_{1}$ and the proof of theorem 2.2 is complete.

Finally, we translate theorem 2.2 in terms of the vorticity $\omega(x, z, t)$ in the original variables:

Corollary 2.3 Let $0<\mu<1 / 2$ and $m>1+2 \mu$. There exists $\epsilon_{0}^{\prime}>0$ such that for all initial data $\omega_{0} \in L^{2}(m)$ with div $\omega_{0}=0$ and $\left\|\omega_{0}\right\|_{m} \leq \epsilon_{0}^{\prime}$, equation (5) has a unique global solution $\omega \in \mathcal{C}^{0}\left([0,+\infty) ; L^{2}(m)\right)$ satisfying $\omega(0)=\omega_{0}$ and div $\omega=0$. In addition, for any $p \in[1,2]$, there exists $\epsilon_{2}>0$ such that

$$
\left\|\omega(t)-\omega_{\text {app }}(t)\right\|_{L^{p}\left(R^{2} \times(0,1)\right)} \leq \frac{\epsilon_{2}}{(1+t)^{1-\frac{1}{p}+\mu}}\left\|\omega_{0}\right\|_{m}, \quad t \geq 0,
$$

where $\omega_{\text {app }}(x, z, t)=\frac{\alpha}{(1+t)} \boldsymbol{G}\left(\frac{x}{\sqrt{1+t}}\right)$ and $\alpha=\int_{R^{2} \times(0,1)}\left(\omega_{0}\right)_{3} d z d x$.

\section{Second-Order Asymptotics}

We now turn our attention to solutions of the integral equation (11) of finite energy, namely when $v(\tau)$ is in $L^{2}\left(\mathbf{R}^{2} \times(0,1)\right)$ for any $\tau \geq 0$. This case can be also characterized by the vorticity since $v(\tau) \in L^{2}\left(\mathbf{R}^{2} \times(0,1)\right)$ is equivalent to $\alpha=\int_{\mathbf{R}^{2} \times(0,1)} w_{3}(\xi, z, \tau) d \xi d z=0$. We showed in section 2 that small solutions of (11) converge to $\alpha \mathbf{G}$ when $\tau$ goes to infinity and we want to precise this behavior when $\alpha=0$. We study the asymptotics in $L^{2}(m)$ for $m>2$. In appendix B.1, we prove that, in $\bar{R}\left(L^{2}(m)\right)$, the discrete spectrum of $\mathcal{L}$ contains at least a simple isolated eigenvalue $\lambda_{0}=0$ with eigenfunction $\mathbf{G}$ and another isolated eigenvalue $\lambda_{1}=-\frac{1}{2}$ of multiplicity 3 with eigenfunctions defined in appendix B.1:

$$
\mathbf{F}_{1}=\left(\begin{array}{c}
0 \\
0 \\
F_{1}
\end{array}\right) ; \mathbf{F}_{2}=\left(\begin{array}{c}
0 \\
0 \\
F_{2}
\end{array}\right) ; \mathbf{F}_{3}=\left(\begin{array}{c}
-F_{2} \\
F_{1} \\
0
\end{array}\right) .
$$

Using these notations and appendix B.3, any solution $w$ of $(11)$ in $L^{2}(m)$ can be decomposed as

$$
\begin{aligned}
w(\xi, z, \tau) & =P_{1} w(\xi, z, \tau)+Q_{1} w(\xi, z, \tau)+\tilde{R} w(\xi, z, \tau) \\
& \equiv \alpha(\tau) \mathbf{G}(\xi)+\sum_{i=1}^{3} \beta_{i}(\tau) \mathbf{F}_{i}(\xi)+q_{1}(\xi, \tau)+r(\xi, z, \tau)
\end{aligned}
$$


where the projections $P_{1}, Q_{1}, \tilde{R}$ and the coefficients $\alpha, \beta_{i}$ are defined in B by $(50,54,56)$. We recall that

$$
\begin{aligned}
\alpha(\tau) & =\int_{\mathbf{R}^{2} \times(0,1)} w_{3}(\xi, z, \tau) d \xi d z \\
\beta_{1}(\tau) & =\int_{\mathbf{R}^{2} \times(0,1)} \xi_{1} w_{3}(\xi, z, \tau) d \xi d z \\
\beta_{2}(\tau) & =\int_{\mathbf{R}^{2} \times(0,1)} \xi_{2} w_{3}(\xi, z, \tau) d \xi d z \\
\beta_{3}(\tau) & =\int_{\mathbf{R}^{2} \times(0,1)} \frac{1}{2}\left(\xi_{1} w_{2}(\xi, z, \tau)-\xi_{2} w_{1}(\xi, z, \tau)\right) d \xi d z .
\end{aligned}
$$

Then, $q_{1}$ belongs to the subspace $\mathcal{W}_{1}$ of $\bar{R}\left(L^{2}(m)\right)$ defined in (55) which is also the spectral subspace associated with the remainder part of the spectrum $\sigma(\mathcal{L})$ in $\bar{R}\left(L^{2}(m)\right)$. In particular,

$$
\int_{\mathbf{R}^{2}} q_{1}(\xi, \tau) d \xi=0, \quad \int_{\mathbf{R}^{2}} \xi_{1} q_{1}(\xi, \tau) d \xi=0, \quad \int_{\mathbf{R}^{2}} \xi_{2} q_{1}(\xi, \tau) d \xi=0 .
$$

Moreover, $\int_{0}^{1} r(\xi, z, \tau) d z=0$ for all $\tau \geq 0$.

The coefficients $\left(\beta_{i}\right)_{i=1, . ., 3}$ satisfy very simple ODE's:

Lemma 3.1 Assume $m>2$ and let $w \in \mathcal{C}^{0}\left([0, T], L^{2}(m)\right)$ be a solution of (11) such that $\alpha=0$. Then, the coefficients $\left(\beta_{i}\right)_{i=1, . ., 3}$ defined by (54) satisfy

$$
\dot{\beta}_{i}(\tau)=-\frac{1}{2} \beta_{i}(\tau), \quad \tau \in[0, T] .
$$

Proof: We write the proof for $i=1$. The two other cases can be proved along the same lines, even for $\beta_{3}(\tau)$ which is defined in a slightly different way.

$$
\dot{\beta}_{1}(\tau)=\int_{\mathbf{R}^{2} \times(0,1)} \xi_{1}\left(\mathcal{L} w_{3}+\sum_{j=1}^{2} \partial_{j}\left(w_{j} v_{3}-v_{j} w_{3}\right)\right) d z d \xi
$$

as $w$ and $v$ are periodic in $z$. Since $\operatorname{div}_{\tau} v(\tau)=\operatorname{div}_{\tau} w(\tau)=0$ and $\operatorname{rot}_{\tau} v(\tau)=$ $w(\tau)$, we find the identities

$$
\begin{aligned}
& \xi_{1} \mathcal{L} w_{3}+\frac{1}{2} \xi_{1} w_{3}=\partial_{1}\left(\xi_{1} \partial_{1} w_{3}+\frac{1}{2} \xi_{1}^{2} w_{3}-w_{3}\right)+\partial_{2}\left(\xi_{1} \partial_{2} w_{3}+\frac{1}{2} \xi_{1} \xi_{2} w_{3}\right) \\
& \int_{\mathbf{R}^{2} \times(0,1)} \xi_{1} \sum_{j=1}^{2} \partial_{j}\left(w_{j} v_{3}-v_{j} w_{3}\right) d z d \xi=-\int_{\mathbf{R}^{2} \times(0,1)} \partial_{2}\left(\frac{v_{1}^{2}+v_{3}^{2}-v_{2}^{2}}{2}\right) d z d \xi .
\end{aligned}
$$

This last equality requires integrations by parts and the fact that $v$ is in $L^{2}\left(\mathbf{R}^{2} \times\right.$ $(0,1))$. Then, $\dot{\beta}_{1}(\tau)=-\frac{1}{2} \beta_{1}(\tau)$.

In particular, it follows from lemma 3.1 that the subspace $\mathcal{W}_{1}$ is invariant under the evolution defined by (11). The remainder $q_{1}$ and $r$ in (23) satisfy the equations:

$$
\begin{array}{rlrl}
\partial_{\tau} q_{1} & =\mathcal{L} q_{1}+Q_{1}(N(w)), \quad \quad \operatorname{div} q_{1} & =0, \xi \in \mathbf{R}^{2}, \tau \geq 0 \\
\partial_{\tau} r & =\Lambda(\tau) r+\tilde{R}(N(w)), \quad \operatorname{div}_{\tau} r(\tau)=0,(\xi, z) \in \mathbf{R}^{2} \times(0,1), \tau \geq 0
\end{array}
$$

where $Q_{1}$ and $\tilde{R}$ are defined in (56) and (50). The following result describes the second order asymptotics of $w(\tau)$ as $\tau$ goes to infinity if $v$ is of finite energy. 
Theorem 3.2 Let $\frac{1}{2}<\nu<1, m>1+2 \nu$. There exists $K_{0}^{\prime \prime}>0$ such that, for all initial data $w_{0} \in L^{2}(m)$ with div $w_{0}=0, \int_{R^{2} \times(0,1)} w_{0} d \xi d z=0$ and $\left\|w_{0}\right\|_{m} \leq$ $K_{0}^{\prime \prime}$, equation (11) has a unique global solution $w \in \mathcal{C}^{0}\left([0,+\infty) ; L^{2}(m)\right)$ satisfying $w(0)=w_{0}$ and for any $\tau \geq 0, \operatorname{div}_{\tau} w(\tau)=0, \int_{\boldsymbol{R}^{2} \times(0,1)} w(\xi, z, \tau) d \xi d z=0$. In addition, there exists $K_{3}>0$ such that

$$
\left\|w(\tau)-\sum_{i=1}^{3} \beta_{i} \boldsymbol{F}_{i} e^{-\frac{\tau}{2}}\right\|_{m} \leq K_{3} e^{-\nu \tau}\left\|w_{0}\right\|_{m}, \quad \tau \geq 0,
$$

where

$$
\begin{aligned}
& \beta_{1}=\int_{\boldsymbol{R}^{2} \times(0,1)} \xi_{1}\left(w_{0}\right)_{3}(\xi, z) d z d \xi, \\
& \beta_{2}=\int_{\boldsymbol{R}^{2} \times(0,1)} \xi_{2}\left(w_{0}\right)_{3}(\xi, z) d z d \xi, \\
& \beta_{3}=\int_{\boldsymbol{R}^{2} \times(0,1)} \frac{1}{2}\left(\xi_{1}\left(w_{0}\right)_{2}-\xi_{2}\left(w_{0}\right)_{1}\right)(\xi, z) d z d \xi .
\end{aligned}
$$

Proof: If $w \in \mathcal{C}^{0}\left([0, \infty) ; L^{2}(m)\right)$ is the solution of (11) given by theorem 1.1 for $K_{0}^{\prime \prime} \leq K_{0}$ and $v$ the corresponding velocity field, we define $\alpha(\tau), \beta_{i}(\tau), q_{1}$ and $r$ as in (54) and (23). By lemma 2.1, $\alpha(\tau)=\alpha(0)=0$ and $v$ is of finite energy for any time. By lemma 3.1, $\dot{\beta}_{i}(\tau)=-\frac{1}{2} \beta_{i}(\tau)$ for $\tau \geq 0$ and $i=1, . ., 3$. Then, $\beta_{i}(\tau)=e^{-\tau / 2} \beta_{i}$ where $\beta_{i}=\beta_{i}(0)$. To bound the remainder terms $q_{1}$ and $r$, we use the integral equations

$$
\begin{aligned}
& q_{1}(\tau)=e^{\tau \mathcal{L}} q_{1}(0)+\int_{0}^{\tau} e^{(\tau-\sigma) \mathcal{L}} Q_{1}(N(w)(\sigma)) d \sigma, \quad \tau \geq 0, \\
& r(\tau)=S(\tau, 0) r(0)+\int_{0}^{\tau} S(\tau, \sigma) \tilde{R}(N(w)(\sigma)) d \sigma, \quad \tau \geq 0 .
\end{aligned}
$$

As far as $q_{1}$ is concerned, we bound $\left\|q_{1}\right\|_{m}$ in three steps.

First step: We easily prove that

$$
\left\|e^{\tau \mathcal{L}} q_{1}(0)\right\|_{m} \leq C e^{-\nu \tau}\left\|w_{0}\right\|_{m} .
$$

Indeed, (26) follows from proposition B.1 with $n=1, q=2, \alpha=0$ and $\epsilon \in(0, m-1-2 \nu)$.

Second step: To bound the integral term in (25), we notice that the moments up to order 1 of $N$ are zero and

$$
Q_{1}(N(w))=Q_{0}(N(w))=\int_{0}^{1} N(w) d z=\left(\sum_{j=1}^{2} \int_{0}^{1} \partial_{j} M_{i j} d z\right)_{i=1, . ., 3} .
$$

We cut (25) into two integrals between 0 and $\tau-1$ and between $\tau-1$ and $\tau$ in order to obtain an optimal decay rate in time. The first term is bounded by proposition B.1 with $n=1, q=2, \alpha=0, \epsilon>0, m \in(2,3]$ and another time with $n=-1, q=\frac{3}{2}, \alpha=(1,0,0)$ or $(0,1,0), \epsilon>0$, and by Jensen's inequality. 
We then get

$$
\begin{aligned}
& \| \int_{0}^{\tau-1} e^{(\tau-\sigma) \mathcal{L}} Q_{1}(N(w)) d \sigma\left\|_{m}=\right\| \int_{0}^{\tau-1} e^{(\tau-\sigma-1) \mathcal{L}} Q_{1} e^{\mathcal{L}} Q_{1}(N(w)) d \sigma \|_{m} \\
& \leq C \int_{0}^{\tau-1} e^{\frac{\tau-\sigma-1}{2}(1-m+\epsilon)}\left\|e^{\mathcal{L}} Q_{1} N(w)\right\|_{m} d \sigma \\
& \leq C \int_{0}^{\tau-1} e^{\frac{\tau-\sigma-1}{2}(1-m+\epsilon)} \sum_{(i, j) \in I}\left\|\partial_{j} e^{\mathcal{L}-\frac{1}{2}} \int_{0}^{1} M_{i j} d z\right\|_{m} d \sigma \\
& \leq C \int_{0}^{\tau-1} e^{\frac{\tau-\sigma}{2}(1-m+\epsilon)}\|w(\sigma)\|_{m}^{2} d \sigma,
\end{aligned}
$$

where $I=\{1,2,3\} \times\{1,2\}$. According to theorem 2.2, for $0<\mu<1 / 2$ and $m>1+2 \mu,\|w(\sigma)\|_{m} \leq K_{2} e^{-\mu \sigma}\left\|w_{0}\right\|_{m}$. The previous term is then bounded by

$$
C K_{2}^{2}\left\|w_{0}\right\|_{m}^{2} e^{-2 \mu \tau} \int_{1}^{\tau} e^{\frac{u}{2}(1-m+\epsilon+4 \mu)} d u
$$

Taking $\nu=2 \mu, m>1+2 \nu$ and $\epsilon \in(0, m-1-2 \nu)$, the second step leads to the following estimate

$$
\left\|\int_{0}^{\tau-1} e^{(\tau-\sigma) \mathcal{L}} Q_{1} N(w) d \sigma\right\|_{m} \leq C e^{-\nu \tau}\left\|w_{0}\right\|_{m}^{2} .
$$

The same arguments are still valid when $m>3$ and proposition B. 1 with $n=1$ and $\gamma=1$ leads to estimate $(27)$ for $\frac{1}{2}<\nu<1$.

Thrid step: In a similar way, the third step can be driven as follows

$$
\begin{aligned}
\left\|\int_{\tau-1}^{\tau} e^{(\tau-\sigma) \mathcal{L}} Q_{1} N(w) d \sigma\right\|_{m} & \leq C \int_{\tau-1}^{\tau} e^{-\frac{\tau-\sigma}{2}} \sum_{(i, j) \in I}\left\|\partial_{j} e^{(\tau-\sigma) \mathcal{L}} \int_{0}^{1} M_{i j} d z\right\|_{m} d \sigma \\
& \leq C \int_{\tau-1}^{\tau} \frac{e^{-\frac{\tau-\sigma}{2}}}{a(\tau-\sigma)^{\frac{2}{3}}}\|w(\sigma)\|_{m}^{2} d \sigma \\
& \leq C K_{2}^{2}\left\|w_{0}\right\|_{m}^{2} e^{-2 \mu \tau} \int_{0}^{1} \frac{e^{-\frac{u}{2}(1-4 \mu)}}{a(u)^{\frac{2}{3}}} d u \\
& \leq C e^{-\nu \tau}\left\|w_{0}\right\|_{m}^{2} .
\end{aligned}
$$

It is clear that the previous bound would not have been sharp for the interval $(0, \tau)$ as $1-4 \mu<0$.

Joining inequalities $(26,27)$ and $(28)$, we prove that for $\nu \in\left(\frac{1}{2}, 1\right)$ and $m>1+2 \nu$, there exists $C_{0}>0$ such that for any $\tau \geq 0$,

$$
\left\|q_{1}(\tau)\right\|_{m} \leq C_{0} e^{-\nu \tau}\left\|w_{0}\right\|_{m} .
$$

To turn to the bound of $\|r(\tau)\|_{m}$, we refer to the proof of theorem 2.2 and the 
previous result on the decreasing of $\left\|q_{1}(\tau)\right\|_{m}$ :

$$
\begin{aligned}
& \|r(\tau)\|_{m} \leq C e^{-4 \pi^{2} e^{\tau}}\left\|w_{0}\right\|_{m} \\
& +C(w) \int_{0}^{\tau} \frac{e^{-\frac{\tau-\sigma}{2}} e^{-4 \pi^{2}\left(e^{\tau}-e^{\sigma}\right)}}{a(\tau-\sigma)^{\frac{2}{3}} a\left(e^{\tau}-e^{\sigma}\right)^{\frac{1}{12}}}\left(e^{-\nu \sigma}\left\|w_{0}\right\|_{m}+\|r(\sigma)\|_{m}\right) d \sigma \\
& +C(w) \int_{0}^{\tau} \frac{e^{\frac{\sigma}{2}} e^{-4 \pi^{2}\left(e^{\tau}-e^{\sigma}\right)}}{a(\tau-\sigma)^{\frac{1}{6}} a\left(e^{\tau}-e^{\sigma}\right)^{\frac{7}{12}}}\left(e^{-\nu \sigma}\left\|w_{0}\right\|_{m}+\|r(\sigma)\|_{m}\right) d \sigma \\
& \leq C e^{-\nu \tau}\left\|w_{0}\right\|_{m}+C C(w) \int_{0}^{\tau} \phi(\tau, \sigma)\|r(\sigma)\|_{m} d \sigma .
\end{aligned}
$$

The last inequality is obtained by appendix D.2 with $(\alpha, \beta, \gamma, \delta)$ equal to $(0, \nu-$ $\left.\frac{1}{2}, \frac{2}{3}, \frac{1}{12}\right)$ and $\left(\frac{1}{2}, \nu, \frac{1}{6}, \frac{7}{12}\right)$. Moreover, $\int_{0}^{\tau} \phi(\tau, \sigma) e^{\nu(\tau-\sigma)} d \sigma$ can be bounded independtly of $\tau$ by appendix D.2 with $(\alpha, \beta, \gamma, \delta)$ equal to $\left(0, \nu-\frac{1}{2}, \frac{2}{3}, \frac{1}{12}\right)$ or $\left(\frac{1}{2}, \nu, \frac{1}{6}, \frac{7}{12}\right)$. Denote $f(\tau)=e^{\nu \tau}\|r(\tau)\|_{m}$. There exist positive constants $C_{1}, C_{2}$ such that for any $T>0$,

$$
\|f\|_{L^{\infty}(0, T)} \leq C_{1}\left\|w_{0}\right\|_{m}+C_{2} C(w)\|f\|_{L^{\infty}(0, T)} .
$$

Since $C(w)$ can be taken as small as we want by choice of appropriate initial data, we take $K_{0}^{\prime \prime}$ such that $\left|C_{2} C(w)\right|<\frac{1}{2}$. Then,

$$
\|r(\tau)\|_{m} \leq 2 C_{1} e^{-\nu \tau}\left\|w_{0}\right\|_{m}
$$

and taking $K_{3}=C_{0}+2 C_{1}$ ends the proof of theorem 3.2.

We now translate this second asymptotics theorem in terms of the original variables:

Corollary 3.3 Let $\frac{1}{2}<\nu<1$ and $m>1+2 \nu$. There exists $\epsilon_{0}^{\prime \prime}>0$ such that for all initial data $\omega_{0} \in L^{2}(m)$ with div $\omega_{0}=0, \int_{R^{2} \times(0,1)} \omega_{0} d z d x=0$ and $\left\|\omega_{0}\right\|_{m} \leq \epsilon_{0}^{\prime \prime}$, equation (5) has a unique global solution $\omega \in \mathcal{C}^{0}\left([0,+\infty) ; L^{2}(m)\right)$ satisfying $\omega(0)=\omega_{0}$ and for any $t \geq 0$, div $\omega(t)=0$ and $\int_{R^{2} \times(0,1)} \omega(t) d z d x=0$. Moreover, for any $p \in[1,2]$, there exists $\epsilon_{3}>0$ such that

$$
\left\|\omega(t)-\omega_{\text {app }}(t)\right\|_{L^{p}\left(\boldsymbol{R}^{2} \times(0,1)\right)} \leq \frac{\epsilon_{3}}{(1+t)^{1-\frac{1}{p}+\nu}}\left\|\omega_{0}\right\|_{m}, \quad t \geq 0
$$

where

$$
\begin{aligned}
& \omega_{\text {app }}(t)=\sum_{i=1}^{3} \frac{\beta_{i}}{(1+t)^{3 / 2}} \boldsymbol{F}_{i}\left(\frac{x}{\sqrt{1+t}}\right) \\
& \beta_{1}=\int_{\boldsymbol{R}^{2} \times(0,1)} x_{1}\left(\omega_{0}\right)_{3} d z d x \\
& \beta_{2}=\int_{\boldsymbol{R}^{2} \times(0,1)} x_{2}\left(\omega_{0}\right)_{3} d z d x \\
& \beta_{3}=\int_{\boldsymbol{R}^{2} \times(0,1)} \frac{1}{2}\left(x_{1}\left(\omega_{0}\right)_{2}-x_{2}\left(\omega_{0}\right)_{1}\right) d z d x .
\end{aligned}
$$




\section{Global Convergence}

We adopt in this section a more general point of view. We are still interested in the asymptotics of bounded global solutions of the integral equation (11) together with periodic boundary conditions (3) but we relax the assumption of smallness of the initial data. Of course, in this more general case, we do not know how to show existence of solutions. However, as $w=\alpha \mathbf{G}$ is such a global bounded solution, we may assume that there exist some of them. Under this assumption, we prove, following [7], that the result of the second section can be generalised. Indeed, the asymptotics of such solutions are still governed by Oseen vortices. The main result of this section can be stated as follows:

Theorem 4.1 Let $m>1$ and $w \in \mathcal{C}^{0}\left([0,+\infty) ; L^{2}(m)\right)$ be a global solution of (11) that is uniformly bounded in time in $L^{2}(m)$. Then,

$$
\lim _{\tau \rightarrow+\infty}\|w(\tau)-\alpha \boldsymbol{G}\|_{m}=0,
$$

where $\alpha=\int_{\boldsymbol{R}^{2} \times(0,1)} w_{3}(\xi, z, 0) d z d \xi$.

Proof: As the proof of this theorem is quite long, we cut it in six lemmas and corollaries. The main idea is to study the $\omega$-limit set of the trajectory $\{w(\tau)\}_{\tau>0}$ and to prove that its elements are two-dimensional (i.e. independent of $z$ ). We are then able to use the result of [7] where it is shown that Oseen vortices are global attractors of any solution of the two-dimensional Navier-Stokes equation with initial conditions in $L_{2 D}^{2}(m)$, (see appendix B). Finally, using a Lyapunov function, we prove that the $\omega$-limit set of $\{w(\tau)\}_{\tau \geq 0}$ is actually reduced to one element: $\alpha \mathbf{G}$. Let us begin with the following lemma:

Lemma 4.2 Let $w \in \mathcal{C}^{0}\left([0,+\infty) ; L^{2}(m)\right)$ be as in theorem 4.1. Then, there exist positive constants $K_{0}, K_{1}$ such that

$$
\begin{aligned}
\|w(\tau)\|_{m} \leq K_{0}, \quad \tau \geq 0 \\
\|\nabla w(\tau)\|_{m} \leq K_{1}, \quad \tau \geq 1 .
\end{aligned}
$$

Proof of lemma 4.2: Inequality (29) holds by assumptions. Regarding inequality (30), we proceed as in lemma 2.1 in [7].

Since $m>1$, we decompose the solution $w$ with the spectral projection $P_{0}$ defined in appendix B.3 by (54):

$$
w(\tau)=\alpha(\tau) \mathbf{G}+f(\xi, z, \tau)
$$

where $\alpha(\tau)=\int_{\mathbf{R}^{2} \times(0,1)} w_{3}(\xi, z, \tau) d z d \xi$ so that $P_{0} f=0$. Then, as shown in lemma 2.1, $\dot{\alpha}(\tau)=0$ and $f$ satisfies the following equation

$$
\partial_{\tau} f=\Lambda(\tau) f+\left(Q_{0}+\tilde{R}\right) N(w)(\tau)
$$

which can be also written in its integral form

$$
f(\tau)=S(\tau, 0) f(0)+\int_{0}^{\tau} S(\tau, \sigma)\left(Q_{0}+\tilde{R}\right) N(w)(\sigma) d \sigma \equiv F_{1}(\tau)+F_{2}(\tau),
$$

where the projection $\tilde{R}$ is defined in (50). 
Lemma 4.3 Let $F_{1}$ and $F_{2}$ be defined as in (31). There exist positive constants $\gamma, C, K_{2}$ such that

$$
\begin{aligned}
\left\|F_{1}(\tau)\right\|_{m} \leq C e^{-\gamma \tau}\|w(0)\|_{m}, & \tau \geq 0 \\
\left\|F_{2}(\tau)\right\|_{m+1} \leq K_{2}, & \tau \geq 0 \\
\left\|\nabla F_{2}(\tau)\right\|_{m+1} \leq K_{2}, & \tau \geq 1
\end{aligned}
$$

Proof of lemma 4.3: Inequality (32) is an easy consequence of propositions C.1 and B.1. Indeed, $F_{1}(\tau)=e^{\tau \mathcal{L}} Q_{0} w(0)+S(\tau, 0) \tilde{R} w(0)$. The first term is bounded by proposition B.1 with $n=0, q=2$ and $\alpha=0$. The second one is bounded by proposition C.1(b) with $\alpha=0$ and $q=2$. To prove (33), notice as in section 2 that for $i=1, . ., 3$,

$$
\left(Q_{0}+\tilde{R}\right) N(w)(\sigma)_{i}=\sum_{j=1}^{2} \partial_{j} \hat{M}_{i j}+e^{\frac{\sigma}{2}} \partial_{z} \hat{M}_{i 3}
$$

where for $(i, j) \in\{1, . ., 3\}^{2}$ and $v^{f}$ the velocity corresponding to $f$

$$
\hat{M}_{i j}=\alpha\left(\mathbf{G}_{j} v_{i}^{f}-\mathbf{G}_{i} v_{j}^{f}+f_{j} v_{i}^{G}-f_{i} v_{j}^{G}\right)+f_{j} v_{i}^{f}-f_{i} v_{j}^{f} .
$$

Then, using proposition C.1 for $q \in\left(\frac{6}{5}, 2\right]$, we get

$$
\begin{aligned}
\left\|F_{2}(\tau)\right\|_{m+1} & \leq C \int_{0}^{\tau} \sum_{(i, j) \in I} \frac{e^{-\frac{\tau-\sigma}{2}}}{a(\tau-\sigma)^{\frac{1}{q}} a\left(e^{\tau}-e^{\sigma}\right)^{\frac{1}{2}\left(\frac{1}{q}-\frac{1}{2}\right)}}\left\|b^{m+1} \hat{M}_{i j}\right\|_{L^{q}} d \sigma \\
& +C \int_{0}^{\tau} \sum_{i=1}^{3} \frac{e^{\frac{\sigma}{2}} e^{-4 \pi^{2}\left(e^{\tau}-e^{\sigma}\right)}}{a(\tau-\sigma)^{\frac{1}{q}-\frac{1}{2}} a\left(e^{\tau}-e^{\sigma}\right)^{\frac{1}{2}+\frac{1}{2}\left(\frac{1}{q}-\frac{1}{2}\right)}}\left\|b^{m+1} \hat{M}_{i 3}\right\|_{L^{q}} d \sigma
\end{aligned}
$$

where $I=\{1,2,3\} \times\{1,2\}$. Bounding $\left\|b^{m+1} \hat{M}_{i j}\right\|_{L^{q}}$ is slightly different from what we did before. Indeed, for any $(i, j) \in\{1, . ., 3\}^{2}$, using Hölder's inequality and the Biot-Savart law, we get for $q=\frac{3}{2}$

$$
\begin{aligned}
\left\|b^{m+1} \alpha \mathbf{G}_{j} v_{i}^{f}\right\|_{L^{\frac{3}{2}}\left(\mathbf{R}^{2} \times(0,1)\right)} & \leq C|\alpha|\|G\|_{m+1}\left\|v^{f}\right\|_{L^{6}\left(\mathbf{R}^{2} \times(0,1)\right)} \\
& \leq C|\alpha|\|G\|_{m+1}\left(\|\tilde{f}\|_{L^{2}\left(\mathbf{R}^{2} \times(0,1)\right)}+\|\bar{f}\|_{L^{\frac{3}{2}}\left(\mathbf{R}^{2}\right)}\right) \\
& \leq C|\alpha|\|G\|_{m+1}\|f\|_{m} \leq C|\alpha|\|G\|_{m+1} K_{0} .
\end{aligned}
$$

Notice that $\tilde{f}$ and $\bar{f}$ are the usual notations defined in appendix A. More easily, for $q=2$,

$$
\left\|b^{m+1} \alpha v_{i}^{G} f_{j}\right\|_{L^{2}\left(\mathbf{R}^{2} \times(0,1)\right)} \leq C|\alpha|\|f\|_{m}\left\|b v^{G}\right\|_{L^{\infty}} \leq C|\alpha|\left\|b v^{G}\right\|_{L^{\infty}} K_{0} .
$$

The last bound uses the idea that, since $f$ has mean value zero, quantities like $v^{f} f$ decay a little bit faster at infinity than $f$ itself. Using Hölder's inequality and splitting $v^{f}$ as in appendix A, we first get for $(i, j) \in\{1, . ., 3\}^{2}$

$$
\begin{aligned}
\left\|b^{m+1} f_{i} v_{j}^{f}\right\|_{L^{q}\left(\mathbf{R}^{2} \times(0,1)\right)} & \leq C\left\|b^{m} f\right\|_{L^{2}\left(\mathbf{R}^{2} \times(0,1)\right)}\left\|b v^{f}\right\|_{L^{\frac{2 q}{2-q}}\left(\mathbf{R}^{2} \times(0,1)\right)} \\
& \leq C\|f\|_{m}\left(\left\|b \bar{v}^{f}\right\|_{L^{\frac{2 q}{2-q}\left(\mathbf{R}^{2}\right)}}+\left\|b \tilde{v}^{f}\right\|_{L^{\frac{2 q}{2-q}}\left(\mathbf{R}^{2} \times(0,1)\right)}\right) .
\end{aligned}
$$


As far as the first part, independent of $z$, is concerned, we use proposition B.1 in [5]. For $q=\frac{2}{m}$ and $m \in(1,2)$, we get

$$
\begin{aligned}
\left\|b \bar{v}^{f}\right\|_{L^{\frac{2 q}{2-q}\left(\mathbf{R}^{2}\right)}} & \leq\left\|b \bar{v}_{3}^{f}\right\|_{L^{\frac{2 q}{2-q}\left(\mathbf{R}^{2}\right)}}+\left\|b\left(\begin{array}{c}
\bar{v}_{1}^{f} \\
\bar{v}_{2}^{f}
\end{array}\right)\right\|_{L^{\frac{2 q}{2-q}\left(\mathbf{R}^{2}\right)}} \\
& \leq C\left(\left\|b^{m}\left(\begin{array}{c}
\bar{f}_{1} \\
\bar{f}_{2}
\end{array}\right)\right\|_{L^{2}\left(\mathbf{R}^{2}\right)}+\left\|b^{m} \bar{f}_{3}\right\|_{L^{2}\left(\mathbf{R}^{2}\right)}\right) \\
& \leq C\|f\|_{m} .
\end{aligned}
$$

For $m$ greater than 2, the embedding $L^{2}(m) \hookrightarrow L^{2}\left(m^{\prime}\right)$ for $m \leq m^{\prime}$ leads to the same conclusion.

For the second part, the Biot-Savart law A.2 helps to conclude for $q \in\left(\frac{6}{5} ; \frac{3}{2}\right]$ and $m>1$,

$$
\begin{aligned}
\left\|b \tilde{v}^{f}\right\|_{L^{\frac{2 q}{2-q}}\left(\mathbf{R}^{2} \times(0,1)\right)} & \leq\left\|b \tilde{v}^{f}\right\|_{H^{1}\left(\mathbf{R}^{2} \times(0,1)\right)} \leq C\left\|\tilde{v}^{f}\right\|_{H^{1}(1)} \\
& \leq C\|\tilde{f}\|_{L^{2}(1)} \leq C\|\tilde{f}\|_{m} \leq C\|f\|_{m} .
\end{aligned}
$$

Finally, $\left\|b^{m+1} f_{i} v_{j}^{f}\right\|_{L^{q}\left(\mathbf{R}^{2} \times(0,1)\right)} \leq C\|f\|_{m}^{2} \leq C K_{0}^{2}$.

Collecting information from the last three steps where we used different values of $q$ for the different terms and using appendix D.2, we get inequality (33) for $\tau \geq 0$. Once more, (34) is due to the regularizing effect and we omit the details.

Corollary 4.4 Let $w \in \mathcal{C}^{0}\left([0,+\infty) ; L^{2}(m)\right)$ be as in theorem 4.1. Then, the trajectory $\{w(\tau)\}_{\tau \geq 0}$ is relatively compact in $L^{2}(m)$.

Proof of corollary 4.4: We write, as in lemma 4.2, $w=\alpha \mathbf{G}+F_{1}+F_{2}$. Observe that, by Rellich's criterion (see [13]), the embedding of $H^{1}(m+1)$ into $L^{2}(m)$ is compact. Since $F_{1}(\tau)$ converges to zero and $F_{2}(\tau)$ is bounded in $H^{1}(m+1)$ for $\tau \geq 1$, we conclude that $\{w(\tau)\}_{\tau \geq 0}$ is relatively compact in $L^{2}(m)$.

Let $m>1$ and $w \in \mathcal{C}^{0}\left([0 ;+\infty) ; L^{2}(m)\right)$ be a global solution of (11) that is uniformly bounded in time in $L^{2}(m)$. Let $\Omega \subset L^{2}(m)$ denote the $\omega$-limit set of this trajectory. By corollary 4.4, we know that $\Omega$ is nonempty, compact and connected. By lemma $4.3, \Omega$ is also bounded in $H^{1}(m+1)$. By a bootstrap argument, we see that $\Omega$ is bounded in $H^{1}\left(m^{\prime}\right)$ for all $m^{\prime}>1$.

Lemma 4.5 Let $w \in \mathcal{C}^{0}\left([0,+\infty) ; L^{2}(m)\right)$ be as in theorem 4.1. Let $\Omega$ be the $\omega$ limit set of $\{w(\tau)\}_{\tau \geq 0}$. Then, the elements of $\Omega$ are independent of $z$. Namely, $\tilde{R}(\Omega)=\{0\}$.

Proof of lemma 4.5: Let $w \in \mathcal{C}^{0}\left([0,+\infty) ; L^{2}(m)\right)$ be a solution of (11) that is uniformly bounded in time in $L^{2}(m)$. We decompose $w$ as in appendix A:

$$
w(\xi, z, \tau)=\bar{w}(\xi, \tau)+r(\xi, z, \tau)
$$

where $\tilde{R} r=r$ for any $\tau \geq 0$. Then, $r$ satisfies the evolution equation

$$
\partial_{\tau} r=\Lambda(\tau) r+\tilde{R}(N(w)(\tau)), \tau \geq 0
$$


which can be written in the integral form:

$$
r(\tau)=S(\tau, 0) r(0)+\int_{0}^{\tau} S(\tau, \sigma) \tilde{R}(N(w)(\sigma)) d \sigma,
$$

see (19) and (21). Since for $i=1, . ., 3$,

$$
\tilde{R}(N(w))_{i}=\sum_{j=1}^{2} \partial_{j} \tilde{R}\left(\mathcal{M}_{i j}\right)+e^{\frac{\sigma}{2}} \partial_{z} \mathcal{M}_{i 3}
$$

where $\mathcal{M}_{i j}=\bar{w}_{j} v_{i}^{r}-v_{j}^{r} \bar{w}_{i}+r_{j} \bar{v}_{i}-\bar{v}_{j} r_{i}+r_{j} v_{i}^{r}-v_{j}^{r} r_{i}$, we get by proposition C.1,

$$
\begin{aligned}
& \|r(\tau)\|_{m} \leq C e^{-4 \pi^{2} e^{\tau}}\left\|w_{0}\right\|_{m} \\
& +C \int_{0}^{\tau}\left(\frac{e^{-\frac{\tau-\sigma}{2}} e^{-4 \pi^{2}\left(e^{\tau}-e^{\sigma}\right)}}{a(\tau-\sigma)^{\frac{2}{3}} a\left(e^{\tau}-e^{\sigma}\right)^{\frac{1}{12}}}+\frac{e^{\frac{\sigma}{2}} e^{-4 \pi^{2}\left(e^{\tau}-e^{\sigma}\right)}}{a(\tau-\sigma)^{\frac{1}{6}} a\left(e^{\tau}-e^{\sigma}\right)^{\frac{7}{12}}}\right) d \sigma
\end{aligned}
$$

as $\|w(\sigma)\|_{m}$ is uniformly bounded in time. According to appendix D.2 with $(\alpha, \beta, \gamma, \delta)$ equal to $\left(0,0, \frac{2}{3}, \frac{1}{12}\right)$ or $\left(\frac{1}{2}, 0, \frac{1}{6}, \frac{7}{12}\right)$, this integral can be uniformly bounded in time and

$$
\|r(\tau)\|_{m} \leq C e^{-\frac{\tau}{3}}, \quad \tau \geq 0 .
$$

Obviously, $\lim _{\tau \rightarrow+\infty}\|w(\tau)-\bar{w}(\tau)\|_{m}=0$ and the $\omega$-limit set $\Omega$ is made of functions independent of $z$. This ends the proof of lemma 4.5.

Lemma 4.6 Let $w \in \mathcal{C}^{0}\left([0,+\infty) ; L^{2}(m)\right)$ be as in theorem 4.1. Let $\Omega$ be the $\omega$ limit set of $\{w(\tau)\}_{\tau \geq 0}$. Then, $\Omega$ is totally invariant under the evolution defined by the autonomous system

$$
\partial_{\tau} \hat{w}=\mathcal{L} \hat{w}+\left(\hat{w} \cdot \nabla_{\xi} \hat{v}-\hat{v} \cdot \nabla_{\xi} \hat{w}\right), \quad \tau \geq 0,
$$

where $\nabla_{\xi}=\left(\partial_{\xi_{1}}, \partial_{\xi_{2}}, 0\right)^{T}, \hat{w}(\xi, \tau): \boldsymbol{R}^{2} \times \boldsymbol{R}^{+} \rightarrow \boldsymbol{R}^{3}$ and $\hat{v}$ is given in terms of $\hat{w}$ via the Biot-Savart law in appendix A.

Remark: If the initial condition $\hat{w}_{0}$ is independent of $z$, this property is preserved by (36) and (36) is nothing but equation (9) applied to functions independent of $z$.

Proof of lemma 4.6: Denote $S_{2 D}(\tau)$ the dynamical system associated with (36). Then, any solution $\hat{w}(\tau)$ of $(36)$ with initial data $\hat{w}_{0}$ is given by $S_{2 D}(\tau) \hat{w}_{0}$. We observe that

$$
S_{2 D}(\tau) \hat{w}_{0}=e^{\tau \mathcal{L}} \hat{w}_{0}+\int_{0}^{\tau} e^{(\tau-\sigma) \mathcal{L}} \hat{N}\left(S_{2 D}(\sigma) \hat{w}_{0}\right) d \sigma
$$

where $\hat{N}(\hat{w})=\left(\hat{w} \cdot \nabla_{\xi}\right) \hat{v}-\left(\hat{v} \cdot \nabla_{\xi}\right) \hat{w}$. We devide this proof in two steps. First, we prove that $\Omega$ is positively invariant (i.e $S_{2 D}(\tau) \Omega \subset \Omega$ ) and then, that $\Omega$ is included in $S_{2 D}(\tau) \Omega$. Thus, we prove that $\Omega$ is totally invariant under the evolution defined by (36).

First step: Let $\hat{w}_{0} \in \Omega$ and $T>0$. We shall prove that $S_{2 D}(\tau) \hat{w}_{0} \in \Omega$ for $\tau \in[0, T]$. By lemma $4.5, \hat{w}_{0}$ is independent of $z$ and there exists $\left(\tau_{n}\right)_{n \in \mathbf{N}}$ such that $\lim _{n \rightarrow+\infty} \tau_{n}=+\infty$ and $\lim _{n \rightarrow+\infty}\left\|w\left(\tau_{n}\right)-\hat{w}_{0}\right\|_{m}=0$. The idea is to show that 
$w\left(\tau+\tau_{n}\right)$ converges to $S_{2 D}(\tau) \hat{w}_{0}$ as $n$ goes to infinity. Therefore, we decompose $w\left(\tau+\tau_{n}\right)$ as in appendix $\mathrm{A}$,

$$
w\left(\tau+\tau_{n}\right)=\bar{w}\left(\tau+\tau_{n}\right)+\tilde{w}\left(\tau+\tau_{n}\right) \equiv \bar{w}_{n}(\tau)+\tilde{w}_{n}(\tau),
$$

and we shall prove that

$$
\begin{aligned}
\left\|\tilde{w}_{n}(\tau)\right\|_{m} & \leq C e^{-\frac{\tau+\tau_{n}}{3}} \\
\left\|\bar{w}_{n}(\tau)-S_{2 D}(\tau) \hat{w}_{0}\right\|_{m} & \leq C \epsilon(n)+C \int_{0}^{\tau}\left\|\bar{w}_{n}(\sigma)-S_{2 D}(\sigma) \hat{w}_{0}\right\|_{m} d \sigma .
\end{aligned}
$$

where $\epsilon(n)$ goes to zero when $n$ goes to infinity. As easily verified, $(38,39)$ and Gronwall's lemma show that

$$
\lim _{n \rightarrow+\infty}\left\|w\left(\tau+\tau_{n}\right)-S_{2 D}(\tau) \hat{w}_{0}\right\|_{m}=0 .
$$

Hence, $S_{2 D}(\tau) \hat{w}_{0} \in \Omega$ and $\Omega$ is positively invariant.

To prove $(38,39)$, notice that $(38)$ is a consequence of $(35)$ while $(39)$ is obtained by combining two integral equations. Indeed, (37) and

$$
\bar{w}_{n}(\tau)=e^{\tau \mathcal{L}} \bar{w}_{n}(0)+\int_{0}^{\tau} e^{(\tau-\sigma) \mathcal{L}} \bar{R} N(w)\left(\tau_{n}+\sigma\right) d \sigma
$$

lead to the following equality

$$
\begin{aligned}
\bar{w}_{n}(\tau)-S_{2 D}(\tau) \hat{w}_{0}= & e^{\tau \mathcal{L}}\left(\bar{w}_{n}(0)-\hat{w}_{0}\right) \\
& +\int_{0}^{\tau} e^{(\tau-\sigma) \mathcal{L}}\left(\bar{R} N(w)\left(\tau_{n}+\sigma\right)-\hat{N}\left(S_{2 D}(\sigma) \hat{w}_{0}\right)\right) d \sigma
\end{aligned}
$$

where

$$
\begin{aligned}
\bar{R} N(w)\left(\tau_{n}+\sigma\right) & =N\left(\bar{w}_{n}\right)(\sigma)+\bar{R} N\left(\tilde{w}_{n}\right)(\sigma) \\
& =N\left(\bar{w}_{n}\right)(\sigma)+\left(\sum_{j=1}^{2} \partial_{j} \int_{0}^{1} \tilde{M}_{i j} d z\right)_{i=1, . .3}
\end{aligned}
$$

and $\tilde{M}_{i j}=\tilde{w}_{j} \tilde{v}_{i}-\tilde{v}_{j} \tilde{w}_{i}$. Using proposition B.1, Jensen's and Hölder's inequalities and the Biot-Savart law, we get for $q \in(1,2)$

$$
\begin{aligned}
\left\|e^{(\tau-\sigma) \mathcal{L}} \bar{R} N\left(\tilde{w}_{n}\right)(\sigma)\right\|_{m} & \leq C \frac{e^{-\frac{\tau-\sigma}{2}}}{a(\tau-\sigma)^{\frac{1}{q}}}\left\|\tilde{w}_{n}\right\|_{m}\left\|\tilde{v}_{n}\right\|_{L^{\frac{2 q}{2-q}}\left(\mathbf{R}^{2} \times(0,1)\right)} \\
& \leq C \frac{e^{-\frac{\tau-\sigma}{2}}}{a(\tau-\sigma)^{\frac{1}{q}}}\left\|\tilde{w}_{n}(\sigma)\right\|_{m}^{2} .
\end{aligned}
$$

Finally,

$$
\left\|\int_{0}^{\tau} e^{(\tau-\sigma) \mathcal{L}} \bar{R} N\left(\tilde{w}_{n}\right)(\sigma) d \sigma\right\|_{m} \leq C e^{-\frac{2 \tau_{n}}{3}}
$$

Then,

$$
\begin{aligned}
\bar{w}_{n}(\tau)- & S_{2 D}(\tau) \hat{w}_{0}=\epsilon(n)+e^{\tau \mathcal{L}}\left(\bar{w}_{n}(0)-\hat{w}_{0}\right) \\
& +\int_{0}^{\tau} e^{(\tau-\sigma) \mathcal{L}}\left(N\left(\bar{w}_{n}\right)(\sigma)-\hat{N}\left(S_{2 D}(\sigma) \hat{w}_{0}\right)\right) d \sigma
\end{aligned}
$$


where $\epsilon(n)$ converges to zero when $n$ goes to infinity. Using proposition B.1 to bound $e^{\tau \mathcal{L}}\left(\bar{w}_{n}(0)-\hat{w}_{0}\right)$ and inequality (14) to bound the integral term, we obtain (39).

Second step: Let $\hat{w}_{0} \in \Omega$. There exists $\left(\tau_{n}\right)_{n \in \mathbf{N}}$ such that $\lim _{n \rightarrow+\infty} \tau_{n}=+\infty$ and $\lim _{n \rightarrow+\infty}\left\|w\left(\tau_{n}\right)-\hat{w}_{0}\right\|_{m}=0$. Let $T>0$. We should prove that $\hat{w}_{0} \in S_{2 D}(T) \Omega$. As $\{w(\tau)\}_{\tau \geq 0}$ is relatively compact, $\left(w\left(\tau_{n}-T\right)\right)_{n \in \mathbf{N}}$ converges towards an element of $\Omega$ denoted $\hat{w}_{-T}$. According to the first step, $w\left(\tau_{n}\right)$ converges in $L^{2}(m)$ to $S_{2 D}(T) \hat{w}_{-T}$. By the uniqueness of such a limit, $\hat{w}_{0}=S_{2 D}(T) \hat{w}_{-T} \in S_{2 D}(T) \Omega$. This ends the proof of lemma 4.6.

Lemma 4.7 Let $w \in \mathcal{C}^{0}\left([0,+\infty) ; L^{2}(m)\right)$ be as in theorem 4.1. Let $\Omega$ be the $\omega$-limit set of $\{w(\tau)\}_{\tau \geq 0}$. Then, $\Omega=\{\alpha \boldsymbol{G}\}, \alpha=\int_{\boldsymbol{R}^{2} \times(0,1)} w_{3}(\xi, z, 0) d \xi d z$.

Proof of lemma 4.7: Let $\hat{w}_{0} \in \Omega$. As $\Omega$ is totally invariant under the evolution defined by (36), there exists a complete trajectory of (36) in $\Omega$ denoted $\{\hat{w}(\tau)\}_{\tau \in \mathbf{R}}$ satisfying $\hat{w}(0)=\hat{w}_{0}$. As $\Omega$ is compact, $\hat{w}(\tau)$ is uniformly bounded in time in $L^{2}(m)$ and by lemma 4.5 , it is independent of $z$. As in appendix A.1, the trajectory divides itself into two independent systems $\left(\hat{w}_{3}, \hat{v}_{1}, \hat{v}_{2}\right)$ and $\left(\hat{w}_{1}, \hat{w}_{2}, \hat{v}_{3}\right)$.

As far as the first system is concerned, $\left\{\hat{w}_{3}(\tau)\right\}$ is a complete trajectory bounded in $L_{2 D}^{2}(m)$ for the evolution studied in [5]. Then, by lemma 3.3 in [7],

$$
\hat{w}_{3}(\tau)=\alpha G \text { and }\left(\hat{v}_{1}, \hat{v}_{2}\right)=\alpha\left(v_{1}^{G}, v_{2}^{G}\right)
$$

where $\alpha=\int_{\mathbf{R}^{2} \times(0,1)} w_{3}(\xi, z, 0) d z d \xi$. For the second system, we look at the velocity $\hat{v}_{3}$ which satisfies

$$
\partial_{\tau} \hat{v}_{3}(\tau)+\left(v_{1}^{G} \partial_{1}+v_{2}^{G} \partial_{2}\right) \hat{v}_{3}(\tau)=\left(\mathcal{L}-\frac{1}{2}\right) \hat{v}_{3}(\tau) .
$$

Since $\hat{w} \in L^{2}(m), m>1$, by lemma A.5, $\hat{v}_{3} \in L^{2}\left(\mathbf{R}^{2}\right)$ and

$$
\frac{1}{2} \frac{d}{d t}\left\|\hat{v}_{3}\right\|_{L^{2}\left(\mathbf{R}^{2}\right)}^{2}=-\int_{\mathbf{R}^{2}}\left|\nabla \hat{v}_{3}\right|^{2} d \xi \leq 0 .
$$

Consider $\Phi(\hat{w})=\int_{\mathbf{R}^{2}}\left|\hat{v}_{3}(\xi)\right|^{2} d \xi$. Then, $\Phi$ is a Lyapunov function for the semiflow defined by (36). More precisely, $\Phi$ is strictly decreasing along the trajectories of (36), except along the subset $\left\{\hat{w} \in L^{2}(m) \mid \hat{v}_{3}=0\right\}$ where $\Phi$ is constant. By LaSalle's invariance principle, the $\omega$-limit and the $\alpha$-limit sets of this trajectory are contained in the set $\left\{\hat{w} \in L^{2}(m) \mid \hat{v}_{3}=0\right\}$. As $\Phi$ must be strictly decreasing or zero along the trajectories,

$$
\left\|\hat{v}_{3}(\tau)\right\|_{L^{2}\left(\mathbf{R}^{2}\right)}=0, \quad \tau \in \mathbf{R} .
$$

As a consequence, $\hat{v}_{3}$ and $\hat{w}_{1}, \hat{w}_{2}$ are zero and

$$
\hat{w}(\tau)=\alpha \mathbf{G}, \tau \in \mathbf{R} .
$$

This implies that $\hat{w}_{0}=\alpha \mathbf{G}$ and concludes the proof of lemma 4.7.

Proof of theorem 4.1: Let $m>1$ and $w \in \mathcal{C}^{0}\left([0,+\infty) ; L^{2}(m)\right)$ be a global solution of (11), uniformly bounded in time in $L^{2}(m)$. By corollary 4.4 and lemma 4.7 , the trajectory $\{w(\tau)\}_{\tau \geq 0}$ is relatively compact and its $\omega$-limit set $\Omega$ is reduced to $\{\alpha \mathbf{G}\}$ where $\alpha=\int_{\mathbf{R}^{2} \times(0,1)} w_{3}(\xi, z, 0) d \xi d z$. This shows that

$$
\lim _{\tau \rightarrow+\infty}\|w(\tau)-\alpha \mathbf{G}\|_{m}=0 .
$$

This ends the proof of theorem 4.1. 


\section{$5 \quad$ Stress-free boundary conditions}

The aim of this section is to rewrite the previous results in the case of stressfree boundary conditions. We consider the vorticity equation (9) together with initial condition $w(\xi, z, 0)=w_{0}(\xi, z)$ and boundary conditions (6). Therefore, we work in the weighted Lebesgue space $L_{s f}^{2}(m)$ defined by

$$
L_{s f}^{2}(m)=\left\{f: \mathbf{R}^{2} \times(0,1) \rightarrow \mathbf{R}^{3} \mid\|f\|_{m}<\infty \text { and } f \text { satisfies }(6)\right\},
$$

where the norm $\|\cdot\|_{m}$ is given in (10).

The main difference that occurs in this case is the splitting $u=\bar{u}+\tilde{u}$ defined in appendix A. Indeed, $\bar{u}$ is here two-dimensional and horizontal and $\tilde{u}$ is no more of mean value zero in $z$, see (47).

Appendix A.3 and A.4 deal with Biot-Savart laws in this case and show that the same estimates hold as for periodic boundary conditions.

As far as appendix B is concerned, the spectrum of the linear operator $\mathcal{L}$ in $\bar{R}\left(L_{s f}^{2}(m)\right)$ is studied in B.5. It is shown that for $m>1$, the discrete spectum of $\mathcal{L}$ in $\bar{R}\left(L_{s f}^{2}(m)\right)$ consists of isolated eigenvalues $\lambda_{k}=-\frac{k}{2}, k \in \mathbf{N}, k<m-1$ with multiplicity $(k+1)$ and that the essential spectrum lies in the half plane $\left\{\lambda \in \mathbf{C} \mid \operatorname{Re}(\lambda) \leq \frac{1-m}{2}\right\}$. Notice that the projection $\bar{R}$, defined in (50), has the same notation as in the periodic case but is slightly different.

Equipped with those results, we are now able to deal with the Cauchy problem (9)-(6) and the asymptotics of solutions.

\subsection{The Cauchy Problem}

Theorem 1.1 can be easily rewritten in $L_{s f}^{2}(m)$. In the Banach space $X$ defined as

$$
X=\left\{w \in \mathcal{C}^{0}\left([0,+\infty) ; L_{s f}^{2}(m)\right) \mid \operatorname{div}_{\tau} w(\tau)=0,\|w\|_{X}=\sup _{\tau \geq 0}\|w(\tau)\|_{m}<+\infty\right\}
$$

estimates (14) still hold and the fixed point theorem leads to the following theorem:

Theorem 5.1 Let $m>1$. There exists $K_{0 s f}>0$ such that, for all initial data $w_{0} \in L_{s f}^{2}(m)$ with div $w_{0}=0$ and $\left\|w_{0}\right\|_{m} \leq K_{0 s f}$, equation (9) has a unique global solution $w \in \mathcal{C}^{0}\left([0,+\infty) ; L_{s f}^{2}(m)\right)$ satisfying $w(0)=w_{0}$ and for any $\tau \geq 0$, $\operatorname{div}_{\tau} w(\tau)=0$. In addition, there exists $K_{1 s f}>0$ such that

$$
\|w(\tau)\|_{m} \leq K_{1 s f}\left\|w_{0}\right\|_{m}, \tau \geq 0 .
$$

\section{$5.2 \quad$ First-Order Asymptotics}

In this section, we determine the first-order asymptotics of solution $w$ given by theorem 5.1. Defining projections $\bar{R}$ and $\tilde{R}$ as in (50) and $P_{0}, Q_{0}$ as in $(54,56)$, we can easily decompose $w$ as

$$
\begin{aligned}
w(\xi, z, \tau) & =P_{0} w(\xi, z, \tau)+Q_{0} w(\xi, z, \tau)+\tilde{R} w(\xi, z, \tau) \\
& =\alpha(\tau) \mathbf{G}(\xi)+q_{0}(\xi, \tau)+r(\xi, z, \tau)
\end{aligned}
$$

The following lemma shows that the conservation of mass still holds: 
Lemma 5.2 Assume $m>1$ and $w \in \mathcal{C}^{0}\left([0, T) ; L_{s f}^{2}(m)\right)$ is a solution of $(9)$. Then, the coefficient $\alpha$ defined in (54) is constant in time.

Proof: As in lemma 2.1, integrating by parts, we get

$$
\begin{aligned}
\dot{\alpha}(\tau) & =\int_{\mathbf{R}^{2} \times(0,1)}\left(\mathcal{L} w_{3}+e^{\tau} \partial_{z}^{2} w_{3}+N(w)_{3}\right) d z d \xi \\
& =\int \nabla_{\xi} \cdot\left(\nabla_{\xi} w_{3}+\frac{1}{2} \xi w_{3}\right)+e^{\tau} \partial_{z}^{2} w_{3}-v_{3} \operatorname{div}_{\tau} w(\tau)+w_{3} \operatorname{div}_{\tau} v(\tau) d z d \xi \\
& =0 .
\end{aligned}
$$

Indeed, $\partial_{z} w_{3}(\xi, 0, \tau)=\partial_{z} w_{3}(\xi, 1, \tau)=0, \operatorname{div}_{\tau} w(\tau)=\operatorname{div}_{\tau} v(\tau)=0$ and $\lim _{|\xi| \rightarrow+\infty} w_{j} v_{i}=0$.

From now on, the proof of theorem 2.2 can be rewritten in the case of stressfree boundary conditions as the estimates satisfied by $q_{0}$ and $r$ are the same as for periodic conditions. It is then straightforward that the following theorem on the first-order asymptotics of solutions holds.

Theorem 5.3 Let $0<\mu<\frac{1}{2}$ and $m>1+2 \mu$. There exists $K_{0 s f}^{\prime}>0$ such that, for all initial data $w_{0} \in L_{s f}^{2}(m)$ with div $w_{0}=0$ and $\left\|w_{0}\right\|_{m} \leq K_{0 s f}^{\prime}$, equation (9) has a unique global solution $w \in \mathcal{C}^{0}\left([0,+\infty) ; L_{s f}^{2}(m)\right)$ satisfying $w(0)=w_{0}$ and for any $\tau \geq 0$, div $v_{\tau} w(\tau)=0$. In addition, there exists $K_{2 s f}>0$ such that

$$
\|w(\tau)-\alpha \boldsymbol{G}\|_{m} \leq K_{2 s f} e^{-\mu \tau}\left\|w_{0}\right\|_{m}, \quad \tau \geq 0,
$$

where $\alpha=\int_{\boldsymbol{R}^{2} \times(0,1)}\left(w_{0}\right)_{3}(\xi, z) d z d \xi$.

\subsection{Second-Order Asymptotics}

For the second-order asymptotics, we can once more rewrite the results of section 3 . With the definitions $(54,56,50)$ of appendix B, we decompose any solution $w \in L_{s f}^{2}(m)$ given by theorem 5.1 as

$$
\begin{aligned}
w(\xi, z, \tau) & =P_{1} w(\xi, z, \tau)+Q_{1} w(\xi, z, \tau)+\tilde{R} w(\xi, z, \tau) \\
& =\alpha(\tau) \mathbf{G}(\xi)+\sum_{i=1}^{2} \beta_{i}(\tau) \mathbf{F}_{i}(\xi)+q_{1}(\xi, \tau)+r(\xi, z, \tau)
\end{aligned}
$$

Looking at solutions of finite energy, $\alpha=0$ and $\dot{\beta}_{i}(\tau)=-\frac{1}{2} \beta_{i}(\tau)$ for $\tau \geq 0$ and $i=1$ or 2 . Therefore, the proof of theorem 3.2 can be easily rewritten in the case of stress-free boundary conditions and we get:

Theorem 5.4 Let $\frac{1}{2}<\nu<1, m>1+2 \nu$. There exists $K_{0 s f}^{\prime \prime}>0$ such that, for all initial data $w_{0} \in L_{s f}^{2}(m)$ with div $w_{0}=0, \int_{\boldsymbol{R}^{2} \times(0,1)} w_{0} d \xi d z=0$ and $\left\|w_{0}\right\|_{m} \leq K_{0 s f}^{\prime \prime}$, equation (9) has a unique global solution

$w \in \mathcal{C}^{0}\left([0,+\infty) ; L_{s f}^{2}(m)\right)$ satisfying $w(0)=w_{0}$ and for any $\tau \geq 0, \operatorname{div}_{\tau} w(\tau)=0$, $\int_{\boldsymbol{R}^{2} \times(0,1)} w(\xi, z, \tau) d \xi d z=0$. In addition, there exists $K_{3 s f}>0$ such that

$$
\left\|w(\tau)-\sum_{i=1}^{2} \beta_{i} \boldsymbol{F}_{i} e^{-\frac{\tau}{2}}\right\|_{m} \leq K_{3 s f} e^{-\nu \tau}\left\|w_{0}\right\|_{m}, \quad \tau \geq 0,
$$

where $\beta_{i}=\int_{R^{2} \times(0,1)} \xi_{i}\left(w_{0}\right)_{3}(\xi, z) d z d \xi$ for $i=1$ or 2 . 
Notice that in the above theorem, the sum $\sum_{i=1}^{2} \beta_{i} \mathbf{F}_{i} e^{-\frac{\tau}{2}}$ only occurs on $i=1$ and 2 . The vector $F_{3}$ does not appear in the asymptotics of solutions for stressfree boundary conditions. Therefore, the long-time behavior of the velocity is, in this case, two-dimensional and horizontal.

\subsection{Global convergence}

As far as global convergence towards Oseen vortices of uniformly bounded solutions of (9) is concerned, we can easily read the proof of theorem 4.1 with appropriate projections $\bar{R}$ and $\tilde{R}$ defined in (50). The same arguments hold as for periodic conditions. A simplification even occurs in lemma 4.7 since the decomposition of appendix A.3 gives directly that $\hat{v}_{3}, \hat{w}_{1}$ and $\hat{w}_{2}$ are zero. A Lyapunov function is in this case useless, (see also the remark at the end of appendix A.5). We thus have the following theorem

Theorem 5.5 Let $m>1$ and $w \in \mathcal{C}^{0}\left([0,+\infty) ; L_{s f}^{2}(m)\right)$ be a global solution of (9) that is uniformly bounded in time in $L_{s f}^{2}(m)$. Then,

$$
\lim _{\tau \rightarrow+\infty}\|w(\tau)-\alpha \boldsymbol{G}\|_{m}=0
$$

where $\alpha=\int_{\boldsymbol{R}^{2} \times(0,1)} w_{3}(\xi, z, 0) d z d \xi$.

\section{A The Biot-Savart Law}

Let $\omega$ be the vorticity given by $\omega(x, z): \mathbf{R}^{3} \rightarrow \mathbf{R}^{3}, 1$-periodic in $z$ and $\operatorname{div} \omega=0$. Define the associated velocity field by $u(x, z): \mathbf{R}^{3} \rightarrow \mathbf{R}^{3}$, 1-periodic in $z$ such that

$$
\left\{\begin{array}{l}
\operatorname{div} u=0, \\
\operatorname{rot} u=\omega .
\end{array}\right.
$$

The aim of this section is to express the velocity field $u$ in terms of the vorticity $\omega$ via the Biot-Savart law and to collect useful estimates for the velocity $u$ in terms of $\omega$. We first decompose the functions $(\omega, u)$ into two parts which still satisfy periodic boundary conditions. The first one $(\bar{\omega}, \bar{u})$ is independent of $z$ and the other one $(\tilde{\omega}, \tilde{u})$ is of mean-value zero in $z$ : for any $(x, z) \in \mathbf{R}^{3}$, we thus set

$$
\begin{gathered}
\omega(x, z)=\bar{\omega}(x)+\tilde{\omega}(x, z), \quad u(x, z)=\bar{u}(x)+\tilde{u}(x, z), \\
\int_{0}^{1} \tilde{\omega}(x, z) d z=0, \quad \int_{0}^{1} \tilde{u}(x, z) d z=0 .
\end{gathered}
$$

Then, $\bar{\omega}=\operatorname{rot} \bar{u}$ and $\tilde{\omega}=\operatorname{rot} \tilde{u}$. Moreover, as $\operatorname{div} \omega=0$ and as $\omega$ is 1-periodic in $z$, notice that $\partial_{1} \bar{\omega}_{1}+\partial_{2} \bar{\omega}_{2}=0$, hence also div $\tilde{\omega}=0$. This means that our decomposition leads to two independent systems with their own Biot-Savart laws:

$$
A .1\left\{\begin{array} { l } 
{ \overline { \omega } , \overline { u } : \mathbf { R } ^ { 2 } \rightarrow \mathbf { R } ^ { 3 } } \\
{ \operatorname { d i v } \overline { \omega } = 0 } \\
{ \operatorname { d i v } \overline { u } = 0 } \\
{ \overline { \omega } = \operatorname { r o t } \overline { u } }
\end{array} \quad A . 2 \left\{\begin{array}{l}
\tilde{\omega}, \tilde{u}: \mathbf{R}^{3} \rightarrow \mathbf{R}^{3}, 1 \text {-periodic in } z \\
\int_{0}^{1} \tilde{\omega}(x, z) d z=\int_{0}^{1} \tilde{u}(x, z) d z=0 \\
\operatorname{div} \tilde{u}=\operatorname{div} \tilde{\omega}=0 \\
\tilde{\omega}=\operatorname{rot} \tilde{u}
\end{array}\right.\right.
$$




\section{A.1 The Biot-Savart law for $(\bar{\omega}, \bar{u})$.}

Let $(\bar{\omega}, \bar{u}): \mathbf{R}^{2} \rightarrow \mathbf{R}^{3}$ such that

$$
\left\{\begin{array}{l}
\partial_{1} \bar{u}_{1}+\partial_{2} \bar{u}_{2}=0 \\
\partial_{1} \bar{\omega}_{1}+\partial_{2} \bar{\omega}_{2}=0 \\
\bar{\omega}=\operatorname{rot} \bar{u}
\end{array}\right.
$$

This system divides itself into two independent systems:

$$
(a)\left\{\begin{array} { l } 
{ \overline { \omega } _ { 1 } = \partial _ { 2 } \overline { u } _ { 3 } } \\
{ \overline { \omega } _ { 2 } = - \partial _ { 1 } \overline { u } _ { 3 } } \\
{ \partial _ { 1 } \overline { \omega } _ { 1 } + \partial _ { 2 } \overline { \omega } _ { 2 } = 0 }
\end{array} \quad ( b ) \left\{\begin{array}{l}
\bar{\omega}_{3}=\partial_{1} \bar{u}_{2}-\partial_{2} \bar{u}_{1} \\
\partial_{1} \bar{u}_{1}+\partial_{2} \bar{u}_{2}=0 .
\end{array}\right.\right.
$$

The second system (b) is equivalent to the Biot-Savart law in $\mathbf{R}^{2}$ (see [5]). Then,

$$
\left(\begin{array}{l}
\bar{u}_{1} \\
\bar{u}_{2}
\end{array}\right)(x)=\frac{1}{2 \pi} \int_{\mathbf{R}^{2}} \frac{(x-y)^{\perp}}{|x-y|^{2}} \bar{\omega}_{3}(y) d y .
$$

If $x=\left(x_{1}, x_{2}\right) \in \mathbf{R}^{2}$, we denote $x^{\perp}=\left(-x_{2}, x_{1}\right)^{T}$. To solve the first system (a), notice that

$$
-\Delta_{x} \bar{u}_{3}=\partial_{1} \bar{\omega}_{2}-\partial_{2} \bar{\omega}_{1} .
$$

By the fundamental solution of the Laplacian $\Delta_{x}$ in $\mathbf{R}^{2}$,

$$
\bar{u}_{3}(x)=-\frac{1}{2 \pi} \int_{\mathbf{R}^{2}} \log (|x-y|)\left(\partial_{1} \bar{\omega}_{2}(y)-\partial_{2} \bar{\omega}_{1}(y)\right) d y,
$$

and integrating by parts, we get

$$
\bar{u}_{3}(x)=-\frac{1}{2 \pi} \int_{\mathbf{R}^{2}} \frac{(x-y)}{|x-y|^{2}} \wedge\left(\begin{array}{c}
\bar{\omega}_{1} \\
\bar{\omega}_{2}
\end{array}\right)(y) d y .
$$

Equalities (43) and (44) are very similar to the Biot-Savart law in $\mathbf{R}^{2}$ and we refer to [17] and [5] for detailed proofs of the following estimates:

Proposition A.1 Let $\bar{u}$ be the velocity field obtained from $\bar{\omega}$ via the Biot-Savart laws (43-44). Assume $1<p<2<q<\infty$ and $\frac{1}{q}=\frac{1}{p}-\frac{1}{2}$. If $\bar{\omega} \in L^{p}\left(\boldsymbol{R}^{2}\right)$, then $\bar{u} \in L^{q}\left(\boldsymbol{R}^{2}\right)$ and there exists $C>0$ such that

$$
\|\bar{u}\|_{L^{q}\left(\boldsymbol{R}^{2}\right)} \leq C\|\bar{\omega}\|_{L^{p}\left(\boldsymbol{R}^{2}\right)} .
$$

\section{A.2 The Biot-Savart law for $(\tilde{\omega}, \tilde{u})$}

Let $(\tilde{\omega}, \tilde{u}): \mathbf{R}^{3} \rightarrow \mathbf{R}^{3}, 1$-periodic in $z$ such that

$$
\left\{\begin{array}{l}
\int_{0}^{1} \tilde{\omega}(x, z) d z=\int_{0}^{1} \tilde{u}(x, z) d z=0 \\
\operatorname{div} \tilde{\omega}=\operatorname{div} \tilde{u}=0 \\
\tilde{\omega}=\operatorname{rot} \tilde{u}
\end{array}\right.
$$

We use a decomposition of $\tilde{\omega}$ and $\tilde{u}$ in Fourier variables $(k, n)$ where our conventions for Fourier transformation are

$$
\begin{aligned}
f(x, z) & =\int_{\mathbf{R}^{2}} \sum_{n \in \mathbf{Z}} f_{n}(k) e^{i(k \cdot x+2 \pi n z)} d k \\
f_{n}(k) & =\frac{1}{2 \pi} \int_{\mathbf{R}^{2}} \int_{0}^{1} f(x, z) e^{-i(k \cdot x+2 \pi n z)} d z d x .
\end{aligned}
$$


Using the relations between the derivatives of $\tilde{u}$ and $\tilde{\omega}$, we easily get for any $k \in \mathbf{R}^{2}$ and any $n \in \mathbf{Z}^{*}$,

$$
\tilde{u}_{n}(k)=\frac{1}{|k|^{2}+4 \pi^{2} n^{2}}\left(\begin{array}{ccc}
0 & -i 2 \pi n & i k_{2} \\
i 2 \pi n & 0 & -i k_{1} \\
-i k_{2} & i k_{1} & 0
\end{array}\right) \tilde{\omega}_{n}(k) .
$$

As in section 1, we shall work in weighted spaces $L^{2}(m)$ defined by (10). The $L^{2}(m)$-norm is also equivalent to another one defined by Fourier coefficients:

$$
\left(\sum_{n \in \mathbf{Z}} \int_{\mathbf{R}^{2}} \sum_{|\alpha| \leq m}\left|\partial^{\alpha} f_{n}(k)\right|^{2} d k\right)^{\frac{1}{2}}
$$

where $\alpha=\left(\alpha_{1}, \alpha_{2}\right) \in \mathbf{N}^{2}, \partial^{\alpha}=\partial_{k_{1}}^{\alpha_{1}} \partial_{k_{2}}^{\alpha_{2}},|\alpha|=\alpha_{1}+\alpha_{2}$. Weighted Sobolev spaces can be defined in a similar way. For instance,

$$
H^{1}(m)=\left\{f \in L^{2}(m) \mid \partial_{i} f \in L^{2}(m), i=1, . ., 3\right\}
$$

and the norm in $H^{1}(m)$ is given by one of the following equivalent expressions:

$$
\begin{aligned}
& \left(\int_{\mathbf{R}^{2}} \int_{0}^{1}\left(1+|x|^{2}\right)^{m}\left(|f(x, z)|^{2}+|\nabla f(x, z)|^{2}\right) d z d x\right)^{\frac{1}{2}}, \\
& \left(\sum_{n \in \mathbf{Z}} \int_{\mathbf{R}^{2}}\left(1+|k|^{2}+n^{2}\right) \sum_{|\alpha| \leq m}\left|\partial^{\alpha} f_{n}(k)\right|^{2} d k\right)^{\frac{1}{2}} .
\end{aligned}
$$

Using these norms and relation (46) where $n$ is different from 0 , we get the following proposition:

Proposition A.2 Let $\tilde{u}$ be the velocity field obtained from $\tilde{\omega}$ via the Biot-Savart law (46). For any $m \in \boldsymbol{N}$, if $\tilde{\omega} \in L^{2}(m)$, then $\tilde{u} \in H^{1}(m)$ and there exists $C>0$ such that

$$
\|\tilde{u}\|_{H^{1}(m)} \leq C\|\tilde{\omega}\|_{L^{2}(m)} .
$$

As a consequence, using Sobolev embedding $H^{1}\left(\mathbf{R}^{2} \times(0,1)\right) \hookrightarrow L^{q}\left(\mathbf{R}^{2} \times(0,1)\right)$ for all $q \in[2,6]$ and proposition A.2 for $m=0$, we get

Corollary A.3 Let $\tilde{u}$ be the velocity field obtained from $\tilde{\omega}$ via the Biot-Savart law (46). If $\tilde{\omega} \in L^{2}\left(\boldsymbol{R}^{2} \times(0,1)\right)$, then $\tilde{u}$ is in $L^{q}\left(\boldsymbol{R}^{2} \times(0,1)\right)$ for any $q \in[2,6]$ and there exists $C>0$ such that

$$
\|\tilde{u}\|_{L^{q}\left(\boldsymbol{R}^{2} \times(0,1)\right)} \leq C\|\tilde{\omega}\|_{L^{2}\left(\boldsymbol{R}^{2} \times(0,1)\right)} .
$$

\section{A.3 The Biot-Savart law in the case of stress-free bound- ary conditions}

Let $\omega(x, z): \mathbf{R}^{2} \times(0,1) \rightarrow \mathbf{R}^{3}$ be the vorticity field which satisfies $\operatorname{div} \omega=0$ and stress-free boundary conditions (6). Define $u(x, z): \mathbf{R}^{2} \times(0,1) \rightarrow \mathbf{R}^{3}$ the corresponding velocity field such that $u$ satisfies stress-free conditions (4) and

$$
\left\{\begin{array}{l}
\operatorname{div} u=0 \\
\operatorname{rot} u=\omega .
\end{array}\right.
$$


We prove in this subsection that the previous Biot-Savart laws stated in appendix A.1 and A.2 still hold with stress-free boundary conditions. To obtain a decomposition of $\omega$ and $u$ which fits with the boundary conditions, we split $\omega$ and $u$ as follows:

$$
\omega(x, z)=\left(\begin{array}{c}
0 \\
0 \\
\bar{\omega}_{3}(x)
\end{array}\right)+\tilde{\omega}(x, z), \quad u(x, z)=\left(\begin{array}{c}
\bar{u}_{1}(x) \\
\bar{u}_{2}(x) \\
0
\end{array}\right)+\tilde{u}(x, z),
$$

where $\tilde{u}_{1}, \tilde{u}_{2}$ and $\tilde{\omega}_{3}$ satisfy Neumann boundary conditions in $z=0$ and $z=1$ and are of mean-value zero in $z$, while $\tilde{\omega}_{1}, \tilde{\omega}_{2}$ and $\tilde{u}_{3}$ satisfy Dirichlet boundary conditions in $z=0$ and $z=1$, see (4) and (6). Notice that the last three functions, $\tilde{\omega}_{1}, \tilde{\omega}_{2}$ and $\tilde{u}_{3}$, are no more of mean-value zero in $z$. We can then divide our problem into two independent systems with their own Biot-Savart laws:

$$
\left\{\begin{array} { l } 
{ \overline { \omega } , \overline { u } : \mathbf { R } ^ { 2 } \rightarrow \mathbf { R } ^ { 3 } } \\
{ \overline { \omega } _ { 1 } = \overline { \omega } _ { 2 } = \overline { u } _ { 3 } = 0 } \\
{ \partial _ { 1 } \overline { u } _ { 1 } + \partial _ { 2 } \overline { u } _ { 2 } = 0 } \\
{ \overline { \omega } _ { 3 } = \partial _ { 1 } \overline { u } _ { 2 } - \partial _ { 2 } \overline { u } _ { 1 } }
\end{array} \quad \left\{\begin{array}{l}
\tilde{\omega}, \tilde{u}: \mathbf{R}^{2} \times(0,1) \rightarrow \mathbf{R}^{3} \\
\operatorname{div} \tilde{u}=\operatorname{div} \tilde{\omega}=0 \\
\tilde{\omega}=\operatorname{rot} \tilde{u} \\
\tilde{u}_{1}, \tilde{u}_{2}, \tilde{\omega}_{3} \text { Neumann and mean value zero in } z \\
\tilde{\omega}_{1}, \tilde{\omega}_{2}, \tilde{u}_{3} \text { Dirichlet }
\end{array}\right.\right.
$$

The first system is exactly system A.1(b) which is solved in (43). Therefore, proposition A.1 still holds.

As far as the second system is concerned, we use a Fourier decomposition which takes into account boundary conditions (4) and (6). Namely, for $i=1$ or 2 ,

$$
\begin{aligned}
& \tilde{\omega}_{i}(x, z)=\int_{\mathbf{R}^{2}} \sum_{n=1}^{\infty} \omega_{i n}(k) e^{i k \cdot x} \sin (n \pi z) d k \\
& \tilde{\omega}_{3}(x, z)=\int_{\mathbf{R}^{2}} \sum_{n=1}^{\infty} \omega_{3 n}(k) e^{i k \cdot x} \cos (n \pi z) d k \\
& \tilde{u}_{i}(x, z)=\int_{\mathbf{R}^{2}} \sum_{n=1}^{\infty} u_{i n}(k) e^{i k \cdot x} \cos (n \pi z) d k \\
& \tilde{u}_{3}(x, z)=\int_{\mathbf{R}^{2}} \sum_{n=1}^{\infty} u_{3 n}(k) e^{i k \cdot x} \sin (n \pi z) d k
\end{aligned}
$$

and the relation $\tilde{\omega}=\operatorname{rot} \tilde{u}$ reads for any $k \in \mathbf{R}^{2}$ and any $n \geq 1$,

$$
\tilde{u}_{n}(k)=\frac{1}{|k|^{2}+\pi^{2} n^{2}}\left(\begin{array}{ccc}
0 & n \pi & i k_{2} \\
-n \pi & 0 & -i k_{1} \\
-i k_{2} & i k_{1} & 0
\end{array}\right) \tilde{\omega}_{n}(k) \text {. }
$$

As this relation is very similar to (46), proposition A.2 and corollary A.3 still hold in $L_{s f}^{2}(m)$ and $H_{s f}^{1}(m)$.

\section{A.4 The Biot-Savart law in scaling variables}

As we work in this article with scaling variables, we translate the above results in terms of $(\xi, z, \tau)$ and $(w, v)$ defined in section 1 by $(7)$. As for any $\tau \geq 0$ and 
any $q \geq 1$,

$$
\|u(t)\|_{L^{q}\left(\mathbf{R}^{2} \times(0,1)\right)}=e^{\tau\left(\frac{1}{q}-\frac{1}{2}\right)}\|v(\tau)\|_{L^{q}\left(\mathbf{R}^{2} \times(0,1)\right)}
$$

and

$$
\|\omega(t)\|_{L^{q}\left(\mathbf{R}^{2} \times(0,1)\right)}=e^{\tau\left(\frac{1}{q}-1\right)}\|w(\tau)\|_{L^{q}\left(\mathbf{R}^{2} \times(0,1)\right)},
$$

the above sections A.1, A.2 and A.3 easily imply the following estimates:

Proposition A.4 Let $v$ be the velocity field obtained from $w$ via the Biot-Savart law. Decompose $v$ and $w$ as in (42) or (47). If $\tilde{w} \in L^{2}\left(\boldsymbol{R}^{2} \times(0,1)\right)$, then $\tilde{v} \in L^{q}\left(\boldsymbol{R}^{2} \times(0,1)\right)$ for any $q \in[2,6]$. If $\bar{w} \in L^{p}\left(\boldsymbol{R}^{2}\right)$, then $\bar{v} \in L^{\frac{2 p}{2-p}}\left(\boldsymbol{R}^{2}\right)$ for any $p \in(1,2)$ and

$$
\begin{aligned}
\|\tilde{v}\|_{L^{q}\left(\boldsymbol{R}^{2} \times(0,1)\right)} & \leq C e^{-\frac{\tau}{q}}\|\tilde{w}\|_{L^{2}\left(\boldsymbol{R}^{2} \times(0,1)\right)} \\
\|\bar{v}\|_{L^{\frac{2 p}{2-p}}\left(\boldsymbol{R}^{2}\right)} & \leq C_{p}\|\bar{w}\|_{L^{p}\left(\boldsymbol{R}^{2}\right)} .
\end{aligned}
$$

\section{A.5 Another bound for the velocity}

For the purpose of section 4, we need some further estimates on the velocity field in scaling variables in case it is independent of the third coordinate $z$.

Lemma A.5 Let $m>1$. If $w \in L^{2}(m)$ satisfies div $w=0$ and $\partial_{z} w=0$, then the corresponding velocity field $v$ given by the Biot-Savart law in appendix A.1 satisfies

$$
v_{3} \in L^{2}\left(\boldsymbol{R}^{2}\right)
$$

Proof: Using appendix A.1, we get

$$
\begin{aligned}
v_{3}(\xi) & =-\frac{1}{2 \pi} \int_{\mathbf{R}^{2}} \frac{(\xi-\eta)}{|\xi-\eta|^{2}} \wedge\left(\begin{array}{c}
w_{1} \\
w_{2}
\end{array}\right)(\eta) d \eta \\
-\Delta_{\xi} v_{3} & =\partial_{1} w_{2}-\partial_{2} w_{1} .
\end{aligned}
$$

By the Biot-Savart law, we obtain that if $w \in L^{q}\left(\mathbf{R}^{2}\right)$ for $q \in(1,2)$, then $v \in L^{\frac{2 q}{2-q}}\left(\mathbf{R}^{2}\right)$. But this result is not sufficient to prove this lemma. The idea is to take benefit from another property satisfied by the vorticity. Indeed, the first moments of $w_{i}$ are zero. This result, together with lemma B.2 in [5], enables us to conclude. We divide this proof in three steps.

First step: We show that the moments of the vorticity are zero. Indeed, $w_{i}=\operatorname{div}\left(\xi_{i} w\right)$ and $\xi_{i} w \in L^{2}(m-1)$. As $m>1, L^{2}(m) \hookrightarrow L^{1}\left(\mathbf{R}^{2} \times(0,1)\right)$ and for all $p \in(1,2), p>\frac{2}{m+1}$, we get easily the continuous embedding $L^{2}(m) \hookrightarrow$ $L^{p}\left(\mathbf{R}^{2} \times(0,1)\right)$. Then, $\xi_{i} w \in L^{p}\left(\mathbf{R}^{2} \times(0,1)\right)$ for $p \in\left(\frac{2}{m}, 2\right)$ and $w_{i} \in L^{1}\left(\mathbf{R}^{2} \times\right.$ $(0,1))$. As $w$ is independent of $z$, this implies for $i=1,2$ that

$$
\int_{\mathbf{R}^{2}} w_{i}(\xi) d \xi=0
$$

Second step: According to (48) and (49),

$$
v_{3}(\xi)=-\frac{1}{2 \pi} \int_{\mathbf{R}^{2}}\left(\frac{\xi-\eta}{|\xi-\eta|^{2}}-\frac{\xi}{|\xi|^{2}}\right) \wedge\left(\begin{array}{c}
w_{1} \\
w_{2}
\end{array}\right)(\eta) d \eta .
$$


For all $(\xi, \eta) \in \mathbf{R}^{2}$ with $\xi \neq 0$ and $\xi \neq \eta$, we have the identity

$$
\frac{\xi_{1}-\eta_{1}}{|\xi-\eta|^{2}}-\frac{\xi_{1}}{|\xi|^{2}}=\frac{1}{|\xi|^{2}|\xi-\eta|^{2}}\left(\left(\xi_{1}-\eta_{1}\right) \xi \cdot \eta+\left(\xi_{2}-\eta_{2}\right) \xi \wedge \eta\right)
$$

where $\xi \cdot \eta=\xi_{1} \eta_{1}+\xi_{2} \eta_{2}$ and $\xi \wedge \eta=\xi_{1} \eta_{2}-\xi_{2} \eta_{1}$. A similar estimate holds for $\frac{\xi_{2}-\eta_{2}}{|\xi-\eta|^{2}}-\frac{\xi_{2}}{|\xi|^{2}}$. Therefore,

$$
\left|v_{3}(\xi)\right| \leq C \int_{\mathbf{R}^{2}} \frac{1}{|\xi||\xi-\eta|}|\eta|\left(\left|w_{1}(\eta)\right|+\left|w_{2}(\eta)\right|\right) d \eta .
$$

Combining this estimate with (48), we obtain

$$
\left|b(\xi) v_{3}(\xi)\right| \leq C \int_{\mathbf{R}^{2}} \frac{1}{|\xi-\eta|}|b(\eta) w(\eta)| d \eta .
$$

Third step: Let $1<m<2$ and $w \in L^{2}(m)$. From lemma B.2 in [5] with $u=\left|b v_{3}\right|$ and $\omega=|b w|$, we get for all $q \in(2,+\infty)$,

$$
\left\|b^{m-1-\frac{2}{q}} b v_{3}\right\|_{L^{q}\left(\mathbf{R}^{2}\right)} \leq C\|w\|_{m} .
$$

Finally, by Hölder's inequality and $q=\frac{2}{m-1}>2$, we get

$$
\left\|v_{3}\right\|_{L^{2}\left(\mathbf{R}^{2}\right)} \leq C\left\|b^{-1}\right\|_{L^{\frac{2 q}{q-2}\left(\mathbf{R}^{2}\right)}}\left\|b v_{3}\right\|_{L^{q}\left(\mathbf{R}^{2}\right)} \leq C\|w\|_{m},
$$

hence $v_{3} \in L^{2}\left(\mathbf{R}^{2}\right)$.

For $m$ greater than $2, L^{2}(m) \hookrightarrow L^{2}\left(m^{\prime}\right)$ for some $m^{\prime} \in(1,2)$ and the previous result ends the proof of lemma A.5.

Remark: This result has no interest in case of stress-free conditions since $w \in L_{s f}^{2}(m)$ and $\partial_{z} w=0$ imply $v_{3}=0$.

\section{B Spectrum of the linear operator $\mathcal{L}$}

In this appendix, we are interested in the spectrum of the linear operator $\mathcal{L}$,

$$
\mathcal{L}=\Delta_{\xi}+\frac{1}{2} \xi \cdot \nabla_{\xi}+1, \xi \in \mathbf{R}^{2} .
$$

A complete study has already been carried out in [5] when $\mathcal{L}$ is applied to scalar functions, namely in weighted $L^{2}$-spaces defined for $m \geq 0$ by

$$
\begin{aligned}
& L_{2 D}^{2}(m)=\left\{w: \mathbf{R}^{2} \rightarrow \mathbf{R},\|w\|_{2 D(m)}<\infty\right\} \\
& \|w\|_{2 D(m)}=\left(\int_{\mathbf{R}^{2}}\left(1+|\xi|^{2}\right)^{m}|w(\xi)|^{2} d \xi\right)^{\frac{1}{2}}=\left\|b^{m} w\right\|_{L^{2}\left(\mathbf{R}^{2}\right)} .
\end{aligned}
$$

We use the same notation $\mathcal{L}$ for the operator applied to scalar or vectorial functions, as for any vectorial function $w$,

$$
\mathcal{L} w=\mathcal{L}\left(\begin{array}{c}
w_{1} \\
w_{2} \\
w_{3}
\end{array}\right)=\left(\begin{array}{c}
\mathcal{L} w_{1} \\
\mathcal{L} w_{2} \\
\mathcal{L} w_{3}
\end{array}\right) .
$$


The spectrum $\sigma(\mathcal{L})$ of $\mathcal{L}$ in $L_{2 D}^{2}(m), m \geq 0$, is

$$
\sigma(\mathcal{L})=\left\{\lambda \in \mathbf{C} \mid \operatorname{Re}(\lambda) \leq \frac{1-m}{2}\right\} \cup\left\{-\frac{k}{2} \mid k \in \mathbf{N}\right\}
$$

Moreover, under the assumption $m>1$, the discrete spectrum of $\mathcal{L}$ in $L_{2 D}^{2}(m)$ consists of isolated eigenvalues $\lambda_{k}=-\frac{k}{2}, k \in \mathbf{N}, k<m-1$, with multiplicity $(k+1)$ and the essential spectrum lies in the half plane $\left\{\lambda \in \mathbf{C} \mid \operatorname{Re}(\lambda) \leq \frac{1-m}{2}\right\}$.

We want to generalize this property for vectorial functions, that is to say, we study the spectrum of $\mathcal{L}$ in the space $L^{2}(m)$ or $L_{s f}^{2}(m)$ of vectorial functions defined respectively by (10) or (40) together with the incompressibility condition (8). As $\mathcal{L}$ only acts on the first two components $\xi \in \mathbf{R}^{2}$, we consider its action on functions independent of $z$. Hence, the first idea is to split the vorticity $w$ into $\bar{w}$ and $\tilde{w}$ as we did in appendix A. Let us define some useful projections:

$$
\begin{array}{ll}
\bar{R}: L^{2}(m) \text { or } L_{s f}^{2}(m) & \longrightarrow L^{2}(m) \text { or } L_{s f}^{2}(m) \\
w & \longmapsto \bar{w} \\
\tilde{R}: \quad L^{2}(m) \text { or } L_{s f}^{2}(m) & \longrightarrow L^{2}(m) \text { or } L_{s f}^{2}(m) \\
w & \longmapsto \tilde{w} .
\end{array}
$$

Then, $\mathbf{1}=\bar{R}+\tilde{R}$ and the projectors $\bar{R}$ and $\tilde{R}$ are well defined by (42) in $L^{2}(m)$ or $(47)$ in $L_{s f}^{2}(m)$ depending on the boundary conditions we consider. Notice that for periodic boundary conditions,

$$
\bar{R}\left(L^{2}(m)\right)=\left\{w \in L^{2}(m) \mid \partial_{z} w=0\right\}
$$

while for stress-free conditions,

$$
\bar{R}\left(L_{s f}^{2}(m)\right)=\left\{w \in L_{s f}^{2}(m) \mid w_{1}=w_{2}=0, \partial_{z} w_{3}=0\right\} .
$$

The incompressibility condition (8) states in those two-dimensional spaces that

$$
\nabla_{\xi} \cdot w_{\xi}=\partial_{1} w_{1}+\partial_{2} w_{2}=0 .
$$

We now want to study the spectrum of $\mathcal{L}$ in $\bar{R}\left(L^{2}(m)\right)$ or $\bar{R}\left(L_{s f}^{2}(m)\right)$. Notice that if $\lambda$ is an eigenvalue of $\mathcal{L}$ with eigenfunction $w=\left(w_{1}, w_{2}, w_{3}\right)^{T}$ in $\bar{R}\left(L^{2}(m)\right)$ or $\bar{R}\left(L_{s f}^{2}(m)\right)$, then for $i \in\{1,2,3\}, w_{i}$ is an eigenfunction of $\mathcal{L}$ in $L_{2 D}^{2}(m)$ with eigenvalue $\lambda$. In the next four subsections, we deal with periodic boundary conditions and we postpone the study of stress-free conditions to appendix B.5.

\section{B.1 The discrete spectrum of $\mathcal{L}$.}

For the purpose of this article, we only turn our attention to the first two eigenvalues. In $L_{2 D}^{2}(m), \lambda_{0}=0$ is a simple eigenvalue of $\mathcal{L}$ with eigenfunction $G(\xi)=\frac{1}{4 \pi} e^{-|\xi|^{2} / 4}$ and $\lambda_{1}=-\frac{1}{2}$ is an eigenvalue of multiplicity 2 with eigenfunctions $F_{1}(\xi)=\frac{\xi_{1}}{2} G(\xi)$ and $F_{2}(\xi)=\frac{\xi_{2}}{2} G(\xi)$. As a consequence, 0 and $-\frac{1}{2}$ are eigenvalues of $\mathcal{L}$ in $\bar{R}\left(L^{2}(m)\right)$ with multiplicity less than 3 and 6 respectively. Among the possible eigenfunctions, we must check which ones are in $\bar{R}\left(L^{2}(m)\right)$ and satisfy the incompressibility condition (51).

As far as the first eigenvalue $\lambda_{0}=0$ is concerned, the only suitable eigenfunction is $\mathbf{G}=\left(\begin{array}{l}0 \\ 0 \\ G\end{array}\right)$ since $\partial_{z} G=0$. Then, $\lambda_{0}=0$ is a simple eigenvalue of $\mathcal{L}$ in $\bar{R}\left(L^{2}(m)\right)$ with eigenfunction $\mathbf{G}$. 
The same arguments are valid for the second eigenvalue $\lambda_{1}=-\frac{1}{2}$. Six vectorial eigenfunctions can be built from $F_{1}$ and $F_{2}$ and we must check which ones are suitable. If $w \in \bar{R}\left(L^{2}(m)\right)$ satisfies (51), it follows for $i=1$ or 2 that

$$
\begin{aligned}
\int_{\mathbf{R}^{2} \times(0,1)} w_{i}(\xi, z) d \xi d z & =\int_{\mathbf{R}^{2} \times(0,1)} \operatorname{div}\left(\xi_{i} w\right) d \xi d z \\
& =\int_{\mathbf{R}^{2}} \xi_{i} w(\xi, 1) d \xi-\int_{\mathbf{R}^{2}} \xi_{i} w(\xi, 0) d \xi=0 .
\end{aligned}
$$

Moreover, for $(i, j) \in\{1,2\}^{2}, \operatorname{div}\left(\xi_{i} \xi_{j} w\right)=\xi_{i} w_{j}+\xi_{j} w_{i}$ and as $\partial_{z} w=0$,

$$
\begin{aligned}
\int_{\mathbf{R}^{2} \times(0,1)} \xi_{1} w_{1}(\xi, z) d \xi d z & =\int_{\mathbf{R}^{2} \times(0,1)} \xi_{2} w_{2}(\xi, z) d \xi d z=0 \\
\int_{\mathbf{R}^{2} \times(0,1)} \xi_{1} w_{2}(\xi, z) d \xi d z & =-\int_{\mathbf{R}^{2} \times(0,1)} \xi_{2} w_{1}(\xi, z) d \xi d z .
\end{aligned}
$$

As $\int_{\mathbf{R}^{2} \times(0,1)} \xi_{1} F_{1} d \xi d z=\int_{\mathbf{R}^{2} \times(0,1)} \xi_{2} F_{2} d \xi d z=1$ and $\int_{\mathbf{R}^{2} \times(0,1)} \xi_{j} F_{i} d \xi d z=0$ for $i \neq j$, the only vectorial eigenfunctions which satisfy the above conditions are

$$
\mathbf{F}_{1}=\left(\begin{array}{l}
0 \\
0 \\
F_{1}
\end{array}\right), \mathbf{F}_{2}=\left(\begin{array}{l}
0 \\
0 \\
F_{2}
\end{array}\right), \mathbf{F}_{3}=\left(\begin{array}{l}
-F_{2} \\
F_{1} \\
0
\end{array}\right) .
$$

Since these three vectors are independent vectors, $\lambda_{1}=-\frac{1}{2}$ is an eigenvalue of $\mathcal{L}$ in $L^{2}(m)$ of multiplicity 3 with eigenfunctions $\mathbf{F}_{1}, \mathbf{F}_{2}$ and $\mathbf{F}_{3}$.

Remark: Even if we do not need for the purpose of this article more information on the discrete spectrum of $\mathcal{L}$ in $\bar{R}\left(L^{2}(m)\right)$, we can state that for any $k \in \mathbf{N},-\frac{k}{2}$ is an eigenvalue of $\mathcal{L}$ in $\bar{R}\left(L^{2}(m)\right)$ with multiplicity $(2 k+1)$. Indeed, $-\frac{k}{2}$ is an eigenvalue of $\mathcal{L}$ in $L_{2 D}^{2}(m)$ with multiplicity $(k+1)$. Hence, there could be a maximum of $3(k+1)$ suitable vectorial eigenfunctions. However, to be in $\bar{R}\left(L^{2}(m)\right)$ and verify the incompressibility condition (51), the vectorial eigenfunctions must satisfy $(k+2)$ relations on moments of order $k$. Indeed,

$$
\int_{\mathbf{R}^{2} \times(0,1)} \xi_{1}^{k} w_{1}(\xi, z) d \xi d z=\int_{\mathbf{R}^{2} \times(0,1)} \xi_{2}^{k} w_{2}(\xi, z) d \xi d z=0
$$

and the $k$ other moments of order $k$ of $w_{1}$ can be expressed by the $k$ other moments of order $k$ of $w_{2}$. Therefore, only $3(k+1)-(k+2)=2 k+1$ vectorial eigenfunctions are suitable.

As a consequence, the spectrum $\sigma(\mathcal{L})$ of $\mathcal{L}$ in $\bar{R}\left(L^{2}(m)\right)$ satisfies

$$
\sigma(\mathcal{L}) \supset\left\{-\frac{k}{2} \mid k \in \mathbf{N}\right\} .
$$

\section{B.2 The essential spectrum of $\mathcal{L}$.}

In [5], it is proved that the essential spectrum of $\mathcal{L}$ in $L_{2 D}^{2}(m)$ lies in the half plane $\left\{\lambda \in \mathbf{C} \mid \operatorname{Re}(\lambda) \leq \frac{1-m}{2}\right\}$. For any $\lambda \in \mathbf{C}$ with $\operatorname{Re}(\lambda)<\frac{1-m}{2}$, there exists $\psi_{\lambda} \in C^{\infty}\left(\mathbf{R}^{2}, \mathbf{R}\right)$ such that $\mathcal{L} \psi_{\lambda}=\lambda \psi_{\lambda}$. Then, for any $\lambda \in \mathbf{C}$ with $\operatorname{Re}(\lambda)<\frac{1-m}{2},\left(0,0, \psi_{\lambda}\right)^{T}$ is a vectorial eigenfunction of $\mathcal{L}$ in $\bar{R}\left(L^{2}(m)\right)$ which 
satisfies the incompressibility condition (51) and since the spectrum of $\mathcal{L}$ is closed,

$$
\sigma(\mathcal{L}) \supset\left\{\lambda \in \mathbf{C} \mid \operatorname{Re}(\lambda) \leq \frac{1-m}{2}\right\} .
$$

\section{B.3 The spectral projections.}

Assume $m \geq 0$. For $n \in\{-1,0,1\}$ and $n+1<m$, we define $P_{n}$ the spectral projection onto the $\sum_{k=0}^{n}(2 k+1)$-dimensional subspace of $\bar{R}\left(L^{2}(m)\right)$ spanned by the eigenfunctions of $\mathcal{L}$ corresponding to the eigenvalues $\left\{-\frac{k}{2} \mid k=0, . ., n\right\}$. For any $w \in \bar{R}\left(L^{2}(m)\right)$,

$$
\begin{aligned}
P_{-1} w & =0 \\
P_{0} w & =\alpha \mathbf{G} \\
P_{1} w & =\alpha \mathbf{G}+\sum_{i=1}^{3} \beta_{i} \mathbf{F}_{i}
\end{aligned}
$$

where

$$
\begin{aligned}
\alpha & =\int_{\mathbf{R}^{2} \times(0,1)} w_{3} d \xi d z \\
\beta_{1} & =\int_{\mathbf{R}^{2} \times(0,1)} \xi_{1} w_{3} d \xi d z \\
\beta_{2} & =\int_{\mathbf{R}^{2} \times(0,1)} \xi_{2} w_{3} d \xi d z \\
\beta_{3} & =\int_{\mathbf{R}^{2} \times(0,1)} \frac{1}{2}\left(\xi_{1} w_{2}-\xi_{2} w_{1}\right) d \xi d z .
\end{aligned}
$$

We also denote $\mathcal{W}_{n}$ the complement of the corresponding spectral subspace

$$
\mathcal{W}_{n}=\left\{w \in \bar{R}\left(L^{2}(m)\right) \mid P_{n} w=0\right\} .
$$

Finally, we also define the complementary spectral projection $Q_{n}$ by

$$
Q_{n}=\bar{R}-P_{n} .
$$

Then, $\mathbf{1}=P_{n}+Q_{n}+\tilde{R}$ in case of periodic boundary conditions with $\tilde{R}$ defined in $(50)$.

\section{B.4 The semigroup $e^{\tau \mathcal{L}}$.}

The operator $\mathcal{L}$ is the generator of a linear semigroup $e^{\tau \mathcal{L}}$ in $\bar{R}\left(L^{2}(m)\right)$ which satisfies the following estimates:

Proposition B.1 Let $n \in\{-1,0,1\}, m>n+1$ and $q \in[1,2]$. For all $\alpha=$ $\left(\alpha_{1}, \alpha_{2}, 0\right) \in N^{2} \times\{0\}$ and all $\epsilon>0$, there exists $C>0$ such that for all $w \in \bar{R}\left(L^{2}(m)\right)$ and all $\tau>0$,

$$
\left\|b^{m} \partial^{\alpha} e^{\tau \mathcal{L}} Q_{n} w\right\|_{L^{2}\left(\boldsymbol{R}^{2}\right)} \leq \frac{C e^{-\gamma \tau}}{a(\tau)^{\frac{1}{q}-\frac{1}{2}+\frac{|\alpha|}{2}}}\left\|b^{m} w\right\|_{L^{q}\left(\boldsymbol{R}^{2}\right)}
$$


where

$$
\begin{aligned}
& a(\tau)=1-e^{-\tau} \\
& \gamma=\frac{m-1-\epsilon}{2} \text { if } n+1<m \leq n+2 \\
& \gamma=\frac{n+1}{2} \text { if } m>n+2 .
\end{aligned}
$$

Proof: We deal separately with the different values of $n \in\{-1,0,1\}$. The idea of this proof is to come back to scalar functions to take benefit of Th. Gallay and C.E. Wayne's work in two dimensions, see [5] and proposition B.2 below. Therefore, we introduce other spectral projections for the scalar and twodimensional case (see Appendix A in [5]). For any $n \in \mathbf{N}$, let $\bar{P}_{n}$ be the spectral projection onto the $\sum_{k=0}^{n}(k+1)$-dimensional subspace of $L_{2 D}^{2}(m)$ spanned by the eigenfunctions of $\mathcal{L}$ corresponding to the eigenvalues $\left\{-\frac{k}{2} \mid k=0, . ., n\right\}$. Notice that the condition $\bar{P}_{n} f=0$ is also equivalent to

$$
\int_{\mathbf{R}^{2}} \xi^{\alpha} f(\xi) d \xi=0 \text { for all } \alpha \in \mathbf{N}^{2} \text { with }|\alpha| \leq n .
$$

For any $n<0$, define $\bar{P}_{n}=0$. Moreover, we denote for any $n \in \mathbf{Z}$

$$
\bar{Q}_{n}=\mathbf{1}-\bar{P}_{n} .
$$

Let $m \geq 0, q \in[1,2], \alpha=\left(\alpha_{1}, \alpha_{2}, 0\right)$ and $\epsilon>0$. Assume $w \in \bar{R}\left(L^{2}(m)\right)$.

Case 1: $n=-1$. Then, $Q_{-1} w=\left(\bar{R}-P_{-1}\right) w=w$ and by proposition B.2, we get

$$
\begin{aligned}
\left\|b^{m} \partial^{\alpha} e^{\tau \mathcal{L}} Q_{-1} w\right\|_{L^{2}(m)} & \leq \sum_{i=1}^{3}\left\|b^{m} \partial^{\alpha} e^{\tau \mathcal{L}} \bar{Q}_{-1} w_{i}\right\|_{L^{2}\left(\mathbf{R}^{2}\right)} \\
& \leq \frac{C e^{-\gamma \tau}}{a(\tau)^{\left(\frac{1}{q}-\frac{1}{2}+\frac{|\alpha|}{2}\right)}} \sum_{i=1}^{3}\left\|b^{m} w_{i}\right\|_{L^{q}\left(\mathbf{R}^{2}\right)} \\
& \leq \frac{C e^{-\gamma \tau}}{a(\tau)^{\left(\frac{1}{q}-\frac{1}{2}+\frac{|\alpha|}{2}\right)}}\left\|b^{m} w\right\|_{L^{q}\left(\mathbf{R}^{2}\right)}
\end{aligned}
$$

where $\gamma$ is defined in the statement of proposition B.1.

Case 2: $n=0$. Then,

$$
Q_{0} w=\left(\bar{R}-P_{0}\right) w=w-\alpha \mathbf{G}=\left(\begin{array}{c}
w_{1} \\
w_{2} \\
w_{3}-\alpha G
\end{array}\right) .
$$

As stressed before in this appendix, $\int_{\mathbf{R}^{2} \times(0,1)} w_{1} d \xi d z=\int_{\mathbf{R}^{2} \times(0,1)} w_{2} d \xi d z=$ $\int_{\mathbf{R}^{2} \times(0,1)}\left(w_{3}-\alpha G\right) d \xi d z=0$. As $w$ is independent of $z$, these equalities precisely state that

$$
\bar{Q}_{0} w_{1}=w_{1}, \bar{Q}_{0} w_{2}=w_{2}, \bar{Q}_{0} w_{3}=w_{3}-\alpha G .
$$

Then,

$$
Q_{0} w=\left(\begin{array}{c}
\bar{Q}_{0} w_{1} \\
\bar{Q}_{0} w_{2} \\
\bar{Q}_{0} w_{3}
\end{array}\right)
$$


and by proposition B.2, we get

$$
\begin{aligned}
\left\|b^{m} \partial^{\alpha} e^{\tau \mathcal{L}} Q_{0} w\right\|_{L^{2}\left(\mathbf{R}^{2}\right)} & \leq \sum_{i=1}^{3}\left\|b^{m} \partial^{\alpha} e^{\tau \mathcal{L}} \bar{Q}_{0} w_{i}\right\|_{L^{2}\left(\mathbf{R}^{2}\right)} \\
& \leq \frac{C e^{-\gamma \tau}}{a(\tau)^{\left(\frac{1}{q}-\frac{1}{2}+\frac{|\alpha|}{2}\right)}}\left\|b^{m} w\right\|_{L^{q}\left(\mathbf{R}^{2}\right)}
\end{aligned}
$$

where $\gamma$ is defined as in proposition B.1.

Case 3: $n=1$. Then,

$$
\begin{aligned}
Q_{1} w & =\left(\bar{R}-P_{1}\right) w=w-\left(\alpha \mathbf{G}+\sum_{i=1}^{3} \beta_{i} \mathbf{F}_{i}\right) \\
& =\left(\begin{array}{c}
w_{1}+\beta_{3} F_{2} \\
w_{2}-\beta_{3} F_{1} \\
w_{3}-\alpha G-\beta_{1} F_{1}-\beta_{2} F_{2}
\end{array}\right) .
\end{aligned}
$$

As $\alpha, \beta_{1}, \beta_{2}$ and $\beta_{3}$ have been chosen as in (54) so that the moments up to order one of $Q_{1} w$ are zero,

$$
Q_{1} w=\left(\begin{array}{c}
\bar{Q}_{1} w_{1} \\
\bar{Q}_{1} w_{2} \\
\bar{Q}_{1} w_{3}
\end{array}\right)
$$

and proposition B.2 applies coordinate by coordinate,

$$
\left\|b^{m} \partial^{\alpha} e^{\tau \mathcal{L}} Q_{1} w\right\|_{L^{2}\left(\mathbf{R}^{2}\right)} \leq \frac{C e^{-\gamma \tau}}{a(\tau)^{\left(\frac{1}{q}-\frac{1}{2}+\frac{|\alpha|}{2}\right)}}\left\|b^{m} w\right\|_{L^{q}\left(\mathbf{R}^{2}\right)} .
$$

Then, proposition B.1 holds for all values of $n \in\{-1,0,1\}$.

For easy reference, we reproduce here the main estimates of the study of $e^{\tau \mathcal{L}}$ in $[5]$.

Proposition B.2 (Th. Gallay and C.E. Wayne) Let $m \geq 0, n \in Z$ and $q \in[1,2]$ such that $n+1<m$. For all $\alpha=\left(\alpha_{1}, \alpha_{2}\right) \in \boldsymbol{N}^{2}$ and all $\epsilon>0$, there exists $C>0$ such that for all $w \in L_{2 D}^{2}(m)$ and all $\tau>0$,

$$
\left\|b^{m} \partial^{\alpha} e^{\tau \mathcal{L}} \bar{Q}_{n} w\right\|_{L^{2}\left(\boldsymbol{R}^{2}\right)} \leq \frac{C e^{-\gamma \tau}}{a(\tau)^{\left(\frac{1}{q}-\frac{1}{2}+\frac{|\alpha|}{2}\right)}}\left\|b^{m} w\right\|_{L^{q}\left(\boldsymbol{R}^{2}\right)}
$$

where

$$
\begin{aligned}
& \gamma=\frac{m-1-\epsilon}{2} \text { if } n+1<m \leq n+2 \\
& \gamma=\frac{n+1}{2} \text { if } m>n+2
\end{aligned}
$$

and where $\bar{Q}_{n}$ is defined in (57).

Proof: If $q=2$, proposition B.2 follows from proposition A.2 in [5]. If $q<2$, and $\tau \in(0,2)$, proposition B.2 follows from proposition A.5 in [5]. If $q<2$ and 
$\tau \geq 2$, using the above results, we get

$$
\begin{aligned}
\left\|b^{m} \partial^{\alpha} e^{\tau \mathcal{L}} \bar{Q}_{n} w\right\|_{L^{2}\left(\mathbf{R}^{2}\right)} & =\left\|b^{m} \partial^{\alpha} e^{(\tau-1) \mathcal{L}} \bar{Q}_{n} e^{\mathcal{L}} \bar{Q}_{n} w\right\|_{L^{2}\left(\mathbf{R}^{2}\right)} \\
& \leq \frac{C e^{-\gamma(\tau-1)}}{a(\tau-1)^{\frac{|\alpha|}{2}}}\left\|b^{m} e^{\mathcal{L}} \bar{Q}_{n} w\right\|_{L^{2}\left(\mathbf{R}^{2}\right)} \\
& \leq \frac{C e^{-\gamma \tau}}{a(\tau-1)^{\frac{|\alpha|}{2}} a(1)^{\left(\frac{1}{q}-\frac{1}{2}\right)}}\left\|b^{m} w\right\|_{L^{q}\left(\mathbf{R}^{2}\right)} \\
& \leq \frac{C e^{-\gamma \tau}}{a(\tau)^{\left(\frac{|\alpha|}{2}+\frac{1}{q}-\frac{1}{2}\right)}}\left\|b^{m} w\right\|_{L^{q}\left(\mathbf{R}^{2}\right)} .
\end{aligned}
$$

This ends the proof of proposition B.2.

Remark: Using proposition B.1, it is easy to complete the study of the spectrum $\sigma(\mathcal{L})$ of $\mathcal{L}$ in $\bar{R}\left(L^{2}(m)\right)$. Let $n \in \mathbf{Z}$ and $m \geq 0$ such that $n+1<m \leq$ $n+2$. Then, $\sigma(\mathcal{L})=\sigma\left(\mathcal{L} P_{n}\right) \cup \sigma\left(\mathcal{L} Q_{n}\right)$. By construction, $\sigma\left(\mathcal{L} P_{n}\right)=\emptyset$ if $n<0$ and $\sigma\left(\mathcal{L} P_{n}\right)=\left\{0,-\frac{1}{2}, . .,-\frac{n}{2}\right\}$ if $n \in \mathbf{N}$. On the other hand, by the Hille-Yosida theorem (see [11]) and proposition B.1, $\sigma\left(\mathcal{L} Q_{n}\right) \subset\left\{\lambda \in \mathbf{C} \mid \operatorname{Re}(\lambda) \leq \frac{1-m}{2}\right\}$. Thus, using (52) and (53),

$$
\sigma(\mathcal{L})=\left\{\lambda \in \mathbf{C} \mid \operatorname{Re}(\lambda) \leq \frac{1-m}{2}\right\} \cup\left\{-\frac{k}{2} \mid k \in \mathbf{N}\right\} .
$$

\section{B.5 Stress-free boundary conditions:}

In an analogous way, we can study the spectrum of $\mathcal{L}$ in $\bar{R}\left(L_{s f}^{2}(m)\right)$ where the projector $\bar{R}$ for stress-free boundary conditions has been defined in (50) and (47). We recall that in this case

$$
\bar{R}\left(L_{s f}^{2}(m)\right)=\left\{w \in L_{s f}^{2}(m) \mid w_{1}=w_{2}=0, \partial_{z} w_{3}=0\right\} .
$$

Then, the study of $\sigma(\mathcal{L})$ in $\bar{R}\left(L_{s f}^{2}(m)\right)$ with stress-free boundary conditions can be brought back to the study of [5] for the two-dimensional Navier-Stokes equation. The discrete spectrum of $\mathcal{L}$ in $\bar{R}\left(L_{s f}^{2}(m)\right), m>1$, consists of isolated eigenvalues $\lambda_{k}=-\frac{k}{2}, k \in \mathbf{N}, k<m-1$, with multiplicity $(k+1)$ and eigenvalues $\left(0,0, \phi_{\alpha}\right)^{T}$ where for any $\alpha \in \mathbf{N}^{2}, \phi_{\alpha} \in \mathcal{S}\left(\mathbf{R}^{n}\right)$ is the Hermite function defined by $\phi_{\alpha}=\partial_{\xi}^{\alpha} G$ and $|\alpha|=k$. Namely, $\phi_{(0,0)}=G, \phi_{(1,0)}=F_{1}, \phi_{(0,1)}=F_{2}$. Moreover, the essential spectrum lies in the half plane $\left\{\lambda \in \mathbf{C} \mid \operatorname{Re}(\lambda) \leq \frac{1-m}{2}\right\}$. We can also define spectral projections as in appendix B.3. If for any $n<0, P_{n}=0$ and for any $n \in \mathbf{N}, P_{n}$ is the spectral projection onto the $\sum_{k=0}^{n}(k+1)$-dimensional subspace of $\bar{R}\left(L_{s f}^{2}(m)\right)$ spanned by the eigenfunctions of $\mathcal{L}$ corresponding to the eigenvalues $\left\{-\frac{k}{2} \mid k=0, . ., n\right\}$, we have for any $w \in \bar{R}\left(L_{s f}^{2}(m)\right)$,

$$
\begin{aligned}
P_{-1} w & =0 \\
P_{0} w & =\alpha \mathbf{G} \\
P_{1} w & =\alpha \mathbf{G}+\sum_{i=1}^{2} \beta_{i} \mathbf{F}_{i},
\end{aligned}
$$

where $\alpha, \beta_{1}$ and $\beta_{2}$ are defined in (54). Then, proposition B.1 still holds since it is an easy consequence of proposition B.2. 


\section{Bounds on the evolution operator $S(\tau, \sigma)$}

In this section, we consider the operator $\Lambda(\tau)$ given for any $\tau \geq 0$ by

$$
\Lambda(\tau)=\mathcal{L}+e^{\tau} \partial_{z}^{2}=\left(\Delta_{\xi}+\frac{1}{2} \xi \cdot \nabla_{\xi}+1\right)+e^{\tau} \partial_{z}^{2}
$$

Since its coefficients depend linearly on the space variables $(\xi, z), \Lambda(\tau)$ becomes a first order differential operator when expressed in the Fourier variables $(k, n)$ defined in (45). Indeed, for any $n \in \mathbf{Z}, k \in \mathbf{R}^{2}$ and $\tau>0$,

$$
(\Lambda(\tau) f)_{n}(k)=-\left(|k|^{2}+\frac{1}{2} k \cdot \nabla_{k}+4 \pi^{2} e^{\tau} n^{2}\right) f_{n}(k) .
$$

Then, $\Lambda(\tau)$ is the generator of a family of evolution operators (or evolution system) $S(\tau, \sigma)$ given for any $0 \leq \sigma \leq \tau$ by

$$
S(\tau, \sigma)=e^{(\tau-\sigma) \mathcal{L}} \circ e^{\left(e^{\tau}-e^{\sigma}\right) \partial_{z}^{2}}=e^{\left(e^{\tau}-e^{\sigma}\right) \partial_{z}^{2}} \circ e^{(\tau-\sigma) \mathcal{L}},
$$

or in Fourier variables $(k, n) \in \mathbf{R}^{2} \times \mathbf{Z}$ by

$$
(S(\tau, \sigma) f)_{n}(k)=e^{-a(\tau-\sigma)|k|^{2}} e^{-4 \pi^{2}\left(e^{\tau}-e^{\sigma}\right) n^{2}} f_{n}\left(k e^{-\frac{\tau-\sigma}{2}}\right),
$$

where $a(\tau)=1-e^{-\tau}$. We refer to Henry [8] chapter 7.1 and Pazy [11] chapter 5 for more information on evolution operators or evolution systems. The aim of this section is to prove the following estimates on the evolution system $S(\tau, \sigma)$ for any $0<\sigma<\tau$ :

Proposition C.1 (a) Fix $m>1$. For all $\alpha=\left(\alpha_{1}, \alpha_{2}, \alpha_{3}\right) \in \boldsymbol{N}^{3}$ and $q \in[1,2]$, there exists $C>0$ such that for all $w \in L^{2}(m)$ or $L_{s f}^{2}(m)$ and all $0<\sigma<\tau$,

$$
\left\|\partial^{\alpha} S(\tau, \sigma) w\right\|_{m} \leq \frac{C}{a(\tau-\sigma)^{\frac{1}{q}-\frac{1}{2}+\frac{\alpha_{1}+\alpha_{2}}{2}} a\left(e^{\tau}-e^{\sigma}\right)^{\frac{1}{2}\left(\frac{1}{q}-\frac{1}{2}\right)+\frac{\alpha_{3}}{2}}}\left\|b^{m} w\right\|_{L^{q}\left(\boldsymbol{R}^{2} \times(0,1)\right)}
$$

where $a(\tau)=1-e^{-\tau}$.

(b) Fix $m>1$. For all $\alpha=\left(\alpha_{1}, \alpha_{2}, \alpha_{3}\right) \in \boldsymbol{N}^{3}$ and $q \in[1,2]$, there exists $C>0$ such that for all $w \in L^{2}(m)$ or $L_{s f}^{2}(m)$ and $0<\sigma<\tau$, assuming $\alpha_{3} \neq 0$ or $\tilde{R} w=w$,

$$
\left\|\partial^{\alpha} S(\tau, \sigma) w\right\|_{m} \leq \frac{C e^{-4 \pi^{2}\left(e^{\tau}-e^{\sigma}\right)}}{a(\tau-\sigma)^{\frac{1}{q}-\frac{1}{2}+\frac{\alpha_{1}+\alpha_{2}}{2}} a\left(e^{\tau}-e^{\sigma}\right)^{\frac{1}{2}\left(\frac{1}{q}-\frac{1}{2}\right)+\frac{\alpha_{3}}{2}}}\left\|b^{m} w\right\|_{L^{q}\left(\boldsymbol{R}^{2} \times(0,1)\right)} .
$$

Remark: $L^{2}(m)$ is defined in $(10), L_{s f}^{2}(m)$ in (40) and $\tilde{R}$ in (50).

Proof: To prove (a), we expand $\partial^{\alpha} S(\tau, \sigma) w$ in Fourier series. In the case of periodic conditions,

$$
\partial^{\alpha} S(\tau, \sigma) w(\xi, z)=\sum_{n \in \mathbf{Z}}(2 i \pi n)^{\alpha_{3}} e^{-4 \pi^{2}\left(e^{\tau}-e^{\sigma}\right) n^{2}} \partial^{\left(\alpha_{1}, \alpha_{2}\right)} e^{(\tau-\sigma) \mathcal{L}} w_{n}(\xi) e^{2 i \pi n z}
$$

where for any $n \in \mathbf{Z}$,

$$
w_{n}(\xi)=\int_{0}^{1} w(\xi, z) e^{-2 i \pi n z} d z
$$


Then, using Parseval's equality, we get

$$
\left\|\partial^{\alpha} S(\tau, \sigma) w\right\|_{m}^{2}=\sum_{n \in \mathbf{Z}}(2 \pi n)^{2 \alpha_{3}} e^{-8 \pi^{2}\left(e^{\tau}-e^{\sigma}\right) n^{2}}\left\|b^{m} \partial^{\left(\alpha_{1}, \alpha_{2}\right)} e^{(\tau-\sigma) \mathcal{L}} w_{n}\right\|_{L^{2}\left(\mathbf{R}^{2}\right)}^{2}
$$

In case of stress-free conditions, Fourier series in sinus and cosinus lead to a similar Parseval's equality. By proposition B.1 with $n=-1, m>1$, for any $\epsilon>0$, any $q \in[1,2]$, there exists $C>0$ independent of $n$ such that

$$
\left\|\partial^{\alpha} S(\tau, \sigma) w\right\|_{m} \leq \frac{C}{a(\tau-\sigma)^{\left(\frac{1}{q}-\frac{1}{2}+\frac{\alpha_{1}+\alpha_{2}}{2}\right)}}\left(\sum_{n \in \mathbf{Z}} g_{n}^{2}\left\|b^{m} w_{n}\right\|_{L^{q}\left(\mathbf{R}^{2}\right)}^{2}\right)^{\frac{1}{2}}
$$

where $g_{n}=(2 \pi n)^{\alpha_{3}} e^{-4 \pi^{2}\left(e^{\tau}-e^{\sigma}\right) n^{2}}$. Finally, using Hölder's inequality, we get

$$
\left\|\partial^{\alpha} S(\tau, \sigma) w\right\|_{m} \leq \frac{C}{a(\tau-\sigma)^{\left(\frac{1}{q}-\frac{1}{2}+\frac{\alpha_{1}+\alpha_{2}}{2}\right)}}\left\|g_{n}\right\|_{l^{p}}\left(\left\|\left(\left\|b^{m} w_{n}\right\|_{L^{q}\left(\mathbf{R}^{2}\right)}\right)\right\|_{l^{q^{\prime}}}\right)
$$

where $p$ and $q^{\prime}$ satisfy the relation $\frac{1}{p}+\frac{1}{q^{\prime}}=\frac{1}{2}$. By appendix D.1 with $\gamma=\alpha_{3} p$ and $A=4 \pi^{2} p\left(e^{\tau}-e^{\sigma}\right)$, there exists $C>0$ such that for any $0<\sigma<\tau$,

$$
\left\|g_{n}\right\|_{l^{p}} \leq \frac{C}{a\left(e^{\tau}-e^{\sigma}\right)^{\frac{1}{2 p}+\frac{\alpha_{3}}{2}}} .
$$

Moreover, Riesz-Thorin's interpolation's theory [12] asserts that if $\frac{1}{q}+\frac{1}{q^{\prime}}=1$,

$$
\|\| b^{m} w_{n}\left\|_{L^{q}\left(\mathbf{R}^{2}\right)}\right\|_{l^{q^{\prime}}} \leq C\left\|b^{m} w\right\|_{L^{q}\left(\mathbf{R}^{2} \times(0,1)\right)} .
$$

Indeed, \|\|$b^{m} w_{n}\left\|_{L^{1}\left(\mathbf{R}^{2}\right)}\right\|_{l^{\infty}} \leq\left\|b^{m} w\right\|_{L^{1}\left(\mathbf{R}^{2} \times(0,1)\right)}$ and by Parseval's equality, \|\|$b^{m} w_{n}\left\|_{L^{2}\left(\mathbf{R}^{2}\right)}\right\|_{l^{2}} \leq\left\|b^{m} w\right\|_{L^{2}\left(\mathbf{R}^{2} \times(0,1)\right)}$. This concludes the proof of (a).

As far as the property (b) is concerned, the only difference appears in the bound of $\left\|g_{n}\right\|_{l^{p}}$ in (59). As $\tilde{R} w=w$ or $\alpha_{3} \neq 0$, the sum (58) over $n \in \mathbf{Z}$ only appears in the proof (b) over $n \neq 0$. Indeed, in case of periodic boundary conditions,

$$
\partial^{\alpha} S(\tau, \sigma) w(\xi, z)=\sum_{n \neq 0} i^{\alpha_{3}} g_{n} \partial^{\left(\alpha_{1}, \alpha_{2}\right)} e^{(\tau-\sigma) \mathcal{L}} w_{n}(\xi) e^{2 i \pi n z}
$$

and the same phenomenon occurs in case of stress-free boundary conditions. Then, by appendix D.1,

$$
\left(\sum_{n \neq 0}\left|g_{n}\right|^{p}\right)^{1 / p} \leq C \frac{e^{-4 \pi^{2}\left(e^{\tau}-e^{\sigma}\right)}}{a\left(e^{\tau}-e^{\sigma}\right)^{\frac{1}{2 p}+\frac{\alpha_{3}}{2}}}
$$

This concludes the proof of (b).

\section{Bounds on integrals and series}

The aim of this technical appendix is to precise the bound of a sum which appears in appendix $\mathrm{C}$ and to give some details in the bound of an integral used quite often throughout this paper. 


\section{D.1 How to bound $\sum_{n \in \mathbf{Z}}|n|^{\gamma} e^{-A n^{2}}$ ?}

Proposition D.1 Let $\gamma$ be a positive constant. There exists $C>0$ such that for any $A>0$,

$$
\begin{aligned}
& S(A) \equiv \sum_{n \in Z}|n|^{\gamma} e^{-A n^{2}} \leq \frac{C}{a(A)^{\frac{\gamma+1}{2}}}, \\
& T(A) \equiv \sum_{n \in Z^{*}}|n|^{\gamma} e^{-A n^{2}} \leq \frac{C e^{-A}}{a(A)^{\frac{\gamma+1}{2}}},
\end{aligned}
$$

where $a(A)=1-e^{-A}$.

Proof: Since the function $S$ is continous on $(0,+\infty)$ and uniformly bounded on $[\epsilon,+\infty)$ for any $\epsilon>0$, the bound on $S(A)$ follows from the computation

$$
\lim _{A \rightarrow 0} A^{\frac{\gamma+1}{2}} S(A)=\int_{\mathbf{R}}|x|^{\gamma} e^{-x^{2}} d x=\Gamma\left(\frac{\gamma+1}{2}\right),
$$

where $\Gamma$ is the Euler function defined by $\Gamma(\alpha)=\int_{0}^{+\infty} t^{\alpha-1} e^{-t} d t$. The bound on $T(A)$ is then an easy consequence of the previous result by a change of index.

\section{D.2 Bound on integrals}

Proposition D.2 Let $(\alpha, \beta, \gamma, \delta) \in\left(\boldsymbol{R}^{+}\right)^{4}$ such that $\gamma+\delta<1$. Then, there exists a positive constant $C>0$ such that for any $t \geq 0$,

$$
I(t) \equiv \int_{0}^{t} \frac{e^{\alpha s} e^{\beta(t-s)} e^{-4 \pi^{2}\left(e^{t}-e^{s}\right)}}{a(t-s)^{\gamma} a\left(e^{t}-e^{s}\right)^{\delta}} d s \leq C e^{(\alpha+\gamma-1) t},
$$

where $a(t)=1-e^{-t}$.

Proof: First note some easy estimates: $e^{t}-e^{s}=e^{t} a(t-s)$ and by the mean-value theorem,

$$
e^{s}(t-s) \leq e^{t}-e^{s} \leq e^{t}(t-s), 0 \leq s \leq t .
$$

According to the properties of function $a$, we divide our study in two steps depending if $t$ is greater or smaller than 1 .

First case: If $t \in[0,1]$, it is sufficient to prove that $I(t)$ is uniformly bounded in time. Since

$$
I(t) \leq C \int_{0}^{t} \frac{d s}{(t-s)^{\gamma+\delta}} \leq C \int_{0}^{1} \frac{d u}{u^{\gamma+\delta}}
$$

and $\gamma+\delta<1$, the first step is finished.

Second case: If $t>1$, we divide the integral $I(t)$ at a critical point $s_{0} \in(0, t)$ such that $e^{t}-e^{s_{0}}=1$. Then, $s_{0}=t+\ln a(t)$. We denote $I_{1}$ and $I_{2}$ the two parts of $I(t)$ obtained by this cut:

$$
\begin{aligned}
& I_{1}(t)=\int_{0}^{s_{0}} \frac{e^{\alpha s} e^{\beta(t-s)} e^{-4 \pi^{2}\left(e^{t}-e^{s}\right)}}{a(t-s)^{\gamma} a\left(e^{t}-e^{s}\right)^{\delta}} d s, \\
& I_{2}(t)=\int_{s_{0}}^{t} \frac{e^{\alpha s} e^{\beta(t-s)} e^{-4 \pi^{2}\left(e^{t}-e^{s}\right)}}{a(t-s)^{\gamma} a\left(e^{t}-e^{s}\right)^{\delta}} d s .
\end{aligned}
$$


We first bound $I_{1}$. With the first easy estimate recalled above, we get

$$
I_{1}=e^{(\beta+\gamma) t} e^{-4 \pi^{2} e^{t}} \int_{0}^{s_{0}} \frac{e^{(\alpha-\beta) s} e^{4 \pi^{2} e^{s}}}{\left(e^{t}-e^{s}\right)^{\gamma} a\left(e^{t}-e^{s}\right)^{\delta}} d s .
$$

Taking into account that $0 \leq s \leq s_{0}$ implies $e^{t}-e^{s} \geq 1$, we have

$$
I_{1} \leq C e^{(\beta+\gamma) t} e^{-4 \pi^{2} e^{t}} \int_{0}^{t} e^{(\alpha-\beta) s} e^{4 \pi^{2} e^{s}} d s .
$$

By a change of variables $r=e^{s}$ and some integrations by parts, we bound the last integral as follows

$$
\int_{0}^{t} e^{(\alpha-\beta) s} e^{4 \pi^{2} e^{s}} d s=\int_{1}^{e^{t}} r^{\alpha-\beta-1} e^{4 \pi^{2} r} d r \leq C e^{(\alpha-\beta-1) t} e^{4 \pi^{2} e^{t}} .
$$

Then, $I_{1}(t) \leq C e^{(\alpha+\gamma-1) t}$ for any $t>1$.

As far as $I_{2}$ is concerned, $s$ is greater than $s_{0}$ and $e^{t}-e^{s}$ and $(t-s)$ are in $[0,1]$. Hence, we get

$$
I_{2} \leq C e^{\alpha t} \int_{s_{0}}^{t} \frac{d s}{a(t-s)^{\gamma} a\left(e^{t}-e^{s}\right)^{\delta}} .
$$

Using once more the first easy estimate and a change of variables, we obtain

$$
I_{2} \leq C e^{(\alpha-\delta) t} \int_{0}^{t-s_{0}} \frac{d u}{u^{\gamma+\delta}} \leq C e^{(\alpha-\delta) t}\left(t-s_{0}\right)^{1-\gamma-\delta} .
$$

As $s_{0}=t+\ln a(t)$, we finally get

$$
I_{2}(t) \leq C e^{(\alpha-\delta) t} e^{-(1-\gamma-\delta) t} .
$$

This completes the proof.

\section{References}

[1] CARPIO, A. Asymptotic behavior for the vorticity equations in dimensions two and three. Comm. Partial Differential Equations 1994, 19(5-6), 827872 .

[2] CARPIO A. Large-time behavior in incompressible Navier-Stokes equations. SIAM J. Math. Anal. 1996, 27(2), 449-475.

[3] Chemin J.y.; DESJARDins B.; Gallagher I.; GRENIER E. Mathematical Analysis of Rotating Fluids, book in preparation.

[4] GIGA Y.; KAMBE T. Large time behavior of the vorticity of the twodimensional viscous flow and its application to vortex formation. Commun. Math. Phys. 1988, 117(4), 549-568.

[5] Gallay T.; Wayne C.E. Invariant Manifolds and the Long-Time Asymptotics of the Navier-Stokes and Vorticity Equations on $\mathbf{R}^{2}$. Arch. Rational Mech. Anal. 2002, 163(3), 209-258. 
[6] GALlay T.; WAYNE C.E. Long-Time Asymptotics of the Navier-Stokes and Vorticity Equations on $\mathbf{R}^{3}$. Phil. Trans. R. Soc. Lond. A 2002, 360(1799), $2155-2188$

[7] Gallay T.; WAYNE C.E. Global stability of vortex solutions of the twodimensional Navier-Stokes equation. preprint 2003.

[8] HENRY D. Geometric Theory of Semilinear Parabolic Equations; Springerverlag: Berlin, 1981; Vol. 840.

[9] KATO T. The Navier-Stokes equation for an incompressible fluid in $\mathbf{R}^{2}$ with a measure as the initial vorticity. Diff. and Integral Equations 1994, 7(3-4), 949-966.

[10] MIYAKAWA T.; SCHONBEK M.E. On optimal decay rates for weak solutions to the Navier-Stokes equations in $\mathbf{R}^{n}$. In Proceedings of Partial Differential Equations and Applications, Olomouc, 1999; Math. Bohem., 2001; 126, 443 455.

[11] PAZY A. Semigroups of Linear Operators and Applications to Partial Differential equations; Springer-Verlag: New York, 1983; Vol. 44.

[12] REED M.; SIMON B. Methods of Modern Mathematical Physics, Vol II: Fourier Analysis, Self-adjointness; Academic press: New York, 1975.

[13] REed M.; SIMON B. Methods of Modern Mathematical Physics, Vol IV: Analysis of Operators; Academic press: New York, 1978.

[14] SCHONBEK M.E. $L^{2}$ decay for weak solutions of the Navier-Stokes equations. Arch. Rational Mech. Anal. 1985, 88(3), 209-222.

[15] SCHONBEK M.E. Large time behaviour of solutions to the Navier-Stokes Equations. Comm. Partial Differential Equations 1986, $11(7)$, 733-763.

[16] SCHONBEK M.E. Lower bounds of rates of decay for solutions to the NavierStokes equations. J. Amer. Math. Soc 1991, 4 (3), 423-449.

[17] STEIN E. Singular integrals and differentiability properties of functions; Princeton University Press: Princeton, N.J., 1970. Princeton Mathematical Series, Vol. 30.

[18] TEMAM R. Some developments on Navier-Stokes equations in the second half of the 20th century. In Development of mathematics 1950-2000; Birkhaüser: Basel, 2000; 1049-1106.

[19] temam R. Navier-Stokes Equations, Theory and Numerical Analysis, 3rd rev. Ed.; North-Holland: Amsterdam, 1984.

[20] WIEGNER M. Decay results for weak solutions of the Navier-Stokes equations on $\mathbf{R}^{n}$. J. Lond. Math. Soc., II Ser. 1987, 35(2), 303-313. 